\title{
14 Von den zwei Wissenschafts-/ Informationsmärkten
}

In diesem Kapitel sollen Transformationsprozesse auf den beiden Informationsmärkten, für die kommerziellen proprietären und für die offenen freien Informationsmärkte ${ }^{55}$ analysiert werden. Dabei konzentrieren sich die folgenden Abschnitte auf die Folgen der sich abzeichnenden Institutionalisierung von Open Access im wissenschaftlichen Publikationsgeschehen, ${ }^{546}$ z. B. mit folgenden Fragen:

(1) Wird die Zuständigkeit der Verlage für das Publizieren im kommerziellen Verwertungsparadigma weiterhin bestehen bleiben?

(2) Werden die Verlage (die Content Provider im weiteren Sinne) sich auch im Open-Access-Publizieren eine ähnliche monopolartige Zuständigkeit für das Publizieren sichern können, wie es lange Zeit für das proprietäre Publizieren der Fall war?

(3) Wird der offene Open-Access-Markt weitgehend aus dem Umfeld der Wissenschaft organisiert und sich damit als der Standard auch der institutionellen und organisatorischen Zuständigkeit für das Publizieren durchsetzen und damit in gewisser Weise eine Renaissance der Learned societies bewirken?

(4) Welche Geschäfts-/Organisations-/Finanzierungsmodelle zeichnen sich auf den Open-Access-Märkten ab?

(5) Welche der beiden Entwicklungsalternativen (2 und 3) sollen/werden von der Politik bzw. den öffentlich finanzierten Wissenschaftsorganisationen mit welchen Argumenten unterstützt?

(6) Werden die Verlage/Content Provider für den Fall, dass die Entwicklung in Richtung der durch (3) angesprochenen Frage geht, sich aus dem engeren Publikationsgeschäft schrittweise zurückziehen und sich neue Marktanteile an den Wissenschaftsmärkten dadurch sichern, dass sie neue Produkte mit informationellen Mehrwerten und neue, direkt auf die Wissensproduktion und deren Verwertung bezogenen Dienstleistungen entwickeln?

(7) Welche Folgen könnten die durch (1)-(6) angesprochenen Fragen (und Antworten) für das Urheberrecht haben?

545 In (Kuhlen 1995) wird dafür plädiert, den Begriff des Marktes nicht auf die kommerzielle Bedeutung, auf die kommerziellen proprietären Informationsmärkte, zu beschränken, sondern „Markt“, in Anknüpfung an das antike Verständnis von Agora, auch auf den Austausch von Wissen auf den offenen freien Informationsmärkten zu beziehen. Diese Informationsanalyse war vor 25 Jahren erstellt worden. Die Märkte haben sich seitdem grundlegend verändert. Die These, dass beide Märkten sich nicht ausschließen müssen, kann weiter aufrecht erhalten bleiben.

546 Vgl. (Hilf/Severiens 2013) Vom Open Access für Dokumente und Daten zu Open Content in der Wissenschaft, S. 387ff; vgl. (Sandberger 2017) Die Zukunft wissenschaftlichen Publizierens. 


\title{
14.1 Zur Kompatibilität der beiden Informationsmärkte
}

Das Zusammenspiel beider Märkte kann konfliktär, aber unter bestimmten Bedingungen auch sich ergänzend sein. Konflikte können dadurch entstehen (vgl. Kap. 3), dass unterschiedliche Leitideen aufeinanderprallen. Das sind hier zum einen Leitideen, die sich in einem früheren (hier analogen) Umfeld entwickelt haben und die sich auf bestimmte Werte bzw. zeitabhängige Interpretationen von Werten stützen. Zum anderen sind das Leitideen, die sich in der Gegenwart in einem weitgehend elektronischen Umfeld an neuen Werten bzw. an einem neuen Verständnis bestehender Werte orientieren. Es ist offensichtlich, dass unterschiedliche Leitideen zu einer unterschiedlichen Verwertung führen: zum einen zu einer kommerziell proprietären Verwertung und zum anderen zu einer offenen freien Verwertung. Daraus entstehen die beiden Informationsmärkte, die kommerziell proprietären und die offenen freien Informationsmärkte ${ }^{547}$

\begin{abstract}
Der kommerziellen Verwertung liegen - etwas holzschnittartig vereinfacht und, wie immer hier, auf Bildung und Wissenschaft bezogen - Werte/Prinzipien wie z. B. privates Eigentum, Verknappung des Zugriffs auf die Wissensobjekte und Profitorientierung. Die Verwertung wird geregelt durch bindende Verträge mit den Urhebern, geschützt durch technische Schutzmaßnahmen, durch Lizenzvereinbarungen, aber eben auch durch das Urheberrecht, das damit faktisch auch Verwerterrecht ist.
\end{abstract}

Der offenen Verwertung durch freie Nutzung - ebenso holzschnittartig vereinfacht und ebenso auf Bildung und Wissenschaft bezogen - liegen Werte/Prinzipien zugrunde wie Teilen, Nachhaltigkeit, und freie uneingeschränkte Nutzung für jedermann (Inklusion). Geschützt wird diese Nutzung über Vereinbarungen wie die durch Open-Access-Erklärungen oder Creative-Commons-Regeln, aber auch durch die Persönlichkeitsrechte des Urheberrechts.

Dass die zweite, die freie Verwertungsform nicht umfassend die erste, kommerzielle Verwertungsform ablöst, zeigt sich darin, dass sich weiterhin ein Informationsmarkt behauptet, auf dem aus der Common Pool Resource (CPR) Wissen ein proprietäres kommerziell verwertbares Wirtschaftsgut wird. Aber es schiebt sich immer mehr auch ein anderer Informationsmarkt in den Vordergrund, bei dem Wissen als ein offenes, frei zu nutzendes Gut ausgetauscht werden kann. Das kann und führt

547 Man beachte, dass wir hier auf das doppelte Verständnis von „Verwertung“ zurückgreifen haben. Kommerzielle Verwertung zielt auf den Gewinn ab; Verwertung in der Wissenschaft bedeutet primär, das produzierte Wissen über Wissensobjekte zu veröffentlichen (und, entsprechend der entwickelten Leitideen) zur Nutzung freizugeben. Ebenso sei daran erinnert, dass hier der Marktbegriff nicht auf die kommerzielle Dimension reduziert wird, sondern im Sinne der antiken agora „Markt“ auch als Forum des freien Austausches von Wissen und Information begriffen wird. 
durchaus zu Konflikten, in denen sich auch die das Urheberrecht bestimmende Politik unterschiedlich positioniert. Das gilt gleichermaßen für die EU wie auch für Deutschland. Die öffentlichen Verlautbarungen der politischen Instanzen zielen, vor allem in den Förderprogrammen, immer mehr auf eine offene Open-Access-Politik ab. Die das Urheberrecht bestimmenden Ausschüsse in der EU-Kommission ${ }^{548}$ sowie der die Urheberrechts-Reformen oft bestimmende Rechtsausschuss im Deutschen Bundestag orientieren sich überwiegend an den bestehenden Organisationformen und Geschäftsmodellen und an den (oft genug wörtlich genommenen) Vorgaben des acquis communitaire der EU. Keineswegs spricht die Politik bezüglich der Regulierung von Wissen und Information mit einer Stimme. Hier die Verfechter der Leitidee von Open Access, dort die Bewahrer der kommerziellen Interessen an der Verwertung. Offensichtlich laufen bei diesem Regulierungsthema die Diskurse der einen unabhängig von den Diskursen der anderen.

Die im Rahmen der Institutionenökonomie entwickelte Commons-Theorie (vgl. FN 96) hat auch die theoretische Grundlage für die mögliche Kompatibilität zwischen proprietärer und offener Informationswirtschaft angeboten. Die Besonderheit des Ostrom'schen Ansatz besteht darin, dass die Umsetzung der CPR in Commons mit einer kommerziellen Verwertung nicht inkompatibel sein muss. Voraussetzung für Kompatibilität der CPR Wissen ist, dass das in Wissensobjekten der Urheber repräsentierte Wissen auch über die von den Verlagen erstellten Informationsobjekte frei verfügbar ist. In der Sprache der Institutionenökonomie formuliert, dass die Transformation der common-pool-basierten Wissensobjekte in nutzbare Informationsobjekte nicht auf Prinzipien von privatem Eigentum, der Verknappung des Zugriffs und der Gewinnmaximierung beruhen muss, sondern auf Werten wie Teilen von Wissen, freier Zugriff, freie Nutzung beruhen kann. Eine solche Informationswirtschaft könnte Teil einer allgemeinen Gemeinwohlökonomie sein. ${ }^{549}$ Diese schließt die kommerzielle Verwertung nicht aus - allerdings nur dann, wenn diese nicht im Widerspruch zu dem gemeinwohlorientierten Interesse an einer freien Verfügbarkeit von Wissen steht.

Eine Gemeinwohlökonomie könnte heute - unter den veränderten Bedingungen der gegenwärtigen IKT - aus und von der Wissenschaft selbst entwickelt werden. Das wäre dann eine andere Form der „Ökonomie der Unknappheit“ (Staab 2019). Wissen und Information gehören zweifellos zu den Gütern, die nicht knapp sind und die sich im Gebrauch nicht verbrauchen. Das dafür erforderliche Knowhow für das Publizieren aus der Wissenschaft selbst wäre heute dafür vorhanden. Zur

548 Wie z. B. bis 2019: Digitaler Binnenmarkt; Arbeitsplätze, Wachstum, Investitionen und Wettbewerbsfähigkeit, Wettbewerb, Wirtschaft und Finanzangelegenheiten, Handel.

549 Vgl. die Arbeiten von (Felber 2018) Die Gemeinwohl-Ökonomie (Felber 2019) This is not economy. 
Umsetzung dieses Knowhow müsste die Öffentlichkeit die entsprechenden Mittel bereitstellen. Daraus entstünde so etwas wie ein natürliches, von der Öffentlichkeit gesichertes Monopol, wie es häufig im Zusammenhang der Wasserwirtschaft gefordert und zuweilen auch realisiert wird. Auch die Verfügung über Wissen, in 4.3 als ,the water of the mind“ bezeichnet, ist für jedermann grundlegend und unverzichtbar. Die Entwicklung ist anders verlaufen. Nach wie vor sind die Wissenschaftsmärkte überwiegend durch die kommerziellen Akteure bestimmt (vgl. 14.2).

Die These der unter den oben beschriebenen Bedingungen möglichen Kompatibilität von kommerzieller Informationswirtschaft mit freier Verfügbarkeit ist dadurch aber nicht widerlegt. Darauf deutet der Trend des Open-Access-Publizierens hin. Verlage akzeptieren immer mehr im Prinzip das Open-Access-Paradigma die Leitidee des freien uneingeschränkten Umgangs mit Wissen und Information (Zugriff und Nutzung). Sie tun dies auch deshalb, weil ihnen nichts anderes übrig bleibt - sonst kommt ihnen ein Großteil der Autoren abhand (vgl. 14.6 Wissenschaft wehrt sich). Sie tun das aber wohl auch, weil sie, wie lange schon und auch weiter die Bibliotheken, eine Finanzierungsgarantie vom Staat erwarten und so ihre Stellung auf den Märkten behaupten können (vgl. dazu die DEAL-Vereinbarungen in 14.8). Gegenwärtig offen ist die Antwort auf die Frage, ob diese öffentliche Finanzierung kommerzieller Leistungen ein nachhaltiges Modell oder nur eine Übergangslösung in Richtung von wissenschaftsautonomen Publikationsformen ist.

Die weiteren Abschnitte dieses Kapitels behandeln die folgenden Themen: In 14.2 wird auf das lange Zeit unproblematische Zusammenspiel von Verlagen, Bibliotheken, Wissenschaftler eingegangen; in 14.3 auf die Konsequenzen der Monopole auf den Wissenschaftsmärkten; in 14.4 werden einige Daten zu den Umsätzen auf den kommerziellen Informationsmärkten zusammengestellt; 14.4.2 geht auf die für Wissenschaft zentralen Zeitschriftenmärkte ein; 14.5 zeigt, dass die kommerziellen Informationsmärkte weitgehend auf einem Geschenkmodell beruhen; in 14.6 werden einige Beispiele dafür gegeben, wie sich Wissenschaft gegen die negativen Folgen der Kommerzialisierung von Wissen und Information wehrt; in 14.7 werden aktuelle Daten zu den Open-Access-Zeitschriftenmärkten angegeben; in 14.8 wird am Beispiel DEAL gezeigt, wie sich gegenwärtig Open Access auf die Transformation der Wissenschaftsmärkte auswirken kann. Das trifft, wie in 14.9 angedeutet, auch für das Publizieren von Büchern zu. All dies legt unter 14.10 den Schluss nahe, dass Open Access der Default des wissenschaftlichen Publizierens wird. In 14.11 werden einige Perspektiven für die freien und kommerziellen Wissenschaftsmärkte ausgelotet, wobei sich sowohl konkurrierende offene und kommerzielle Open-Access-Märkte entwickeln könnten als auch neue kommerzielle Märkte mit informationellen Mehrwertprodukten und -dienstleistungen jenseits des Publizierens. 


\subsection{Verlage, Bibliotheken, Wissenschaft - lange Zeit ein unproblematisches Zusammenspiel}

Bis Mitte des 20 Jahrhunderts wurde nicht als problematisch empfunden das $\mathrm{Zu}$ sammenspiel ${ }^{550}$ von a) Wissen produzierenden und nutzenden Akteuren in Bildung und Wissenschaft, b) von verwertenden Akteuren in der Wirtschaft (Verlagen) und c) den öffentlich finanzierten Bibliotheken, welche die Informationsprodukte von den Akteuren unter (b) erworben und den unter (a) angesprochenen Akteuren i. d. R. unentgeltlich zur Verfügung gestellt haben. Die Arbeitsteilung zwischen Produktion, Vertrieb, Vermittlung und Nutzung hat also unter analogen Bedingungen lange Zeit zufriedenstellend funktioniert - wenn auch zuweilen etwas mühsam und zeitraubend über die Fernleihe, wenn die eigene Bibliothek das gewünschte Objekte nicht in ihrem Bestand hatte. Bibliotheken und Verlage zogen lange Zeit sozusagen an einem Strang. Lange Zeit konnten die Bibliotheken die von ihren Wissenschaftlern benötigten Materialien (Bücher und Zeitschriften) weitgehend vollständig zur Verfügung stellen - bei Büchern über Kauf, bei Zeitschriften weitgehend über Lizenzvereinbarungen/Subskriptionen von Zeitschriften. Wie selbstverständlich waren Akteure in Bildung und Wissenschaft in der Lage, das in Informationsprodukten aufbereitete und veröffentlichte Wissen frei über ihre jeweilige Bibliothek zu nutzen. Dem stand auch das Urheberrecht nicht im Wege.

Den Verlagen wurde nicht bestritten, dass sie quasi das technische, fachliche und organisatorische Knowhow-Monopol für die erforderlichen Leistungen besaßen: für das redaktionelle Lektorat, die Organisation der Qualitätssicherung, die Drucklegung, die Erstellung von Exemplaren entsprechend der Auflage, das Marketing, deren Verteilung über den Versand und die Versorgung der Bibliotheken. Diese Leistungen verursachen Kosten, die die Verlage zunächst vorstrecken konnten und die sich über Verkauf oder Lizenz, zusammen mit dem erwarteten Gewinn, im Preis widerspiegeln. Auch das wurde weitgehend von den Bibliotheken als wichtigsten Abnehmern der Informationsprodukte akzeptiert. Die Öffentlichkeit finanzierte dies. ${ }^{551}$ Verlage konnten sich auch noch zu Zeiten, in denen sich die analoge Publikationswelt in eine elektronischen wandelte, als Partner der Wissenschaft behaupten und konnten somit auch auf breite Akzeptanz aus der Wissenschaft zählen. Auch in der Gegenwart gilt das weiter insbesondere für die

550 Zum Zusammenspiel von Verlage und Wissenschaft bzw. zur Transformation des Publizierens nach dem Zweiten Weltkrieg vgl. (Fyfe et al. 2017) Untangling academic publishing. 551 Vgl. die Daten dazu in (Pflüger 2016) Die Bildungs- und Wissenschaftsschranke. 
vielen kleineren Verlage, die, vor allem auf dem Buchmarkt, attraktive Produkte zusammen mit Wissenschaftlern entwickeln.

Für Selbstorganisationsformen aus der Wissenschaft bestand wenig Bedarf, zumal die Hürde, die für das Publizieren nötige technische Infrastruktur aufzubauen, noch bis vor wenigen Jahren sehr hoch war. Wissenschaftler wollen sich mehr um neue Forschung kümmern wollen als abgeschlossene Forschung selbst zu publizieren. Zudem sichern attraktive und prestigehaltige Tätigkeiten für kommerzielle Informationsprodukte (Herausgeber, Mitglied in Beiräten, Gutachter) etablierten Wissenschaftlern Einfluss in ihren Fachgebieten. Vor allem sorgt der von Verlagen organisierte Impact Factor (IF) für starke Bindungen an das bestehende Verlagssystem und für eine vermeintlich objektive Qualitätshierarchie bei den Zeitschriften (vgl. 14.3.2). All das beeinflusst auch heute noch das Publikationsverhalten. Der kommerzielle Wissenschaftsmarkt, vor allem das große Segment, dass sich auf das Publizieren von textuellen Materialien konzentrierte, ${ }^{552}$ war so auch schon zu analogen Zeiten ein einträgliches und vor allem sicheres Geschäft für die Anbieter. Wegen der i. d. R. hohen Preise und Lizenzen für Bücher und Abonnements für Zeitschriften war dieser Markt nur geringfügig ein Endnutzermarkt, sondern ein institutionalisierter Markt mit den Vermittlungsorganisationen wie Bibliotheken als verlässliche und kalkulierbare Partner. ${ }^{553}$ Eine Ausnahme waren Lehr- und Schulbücher, welche zwar auch von den Bibliotheken erworben werden, sogar mit Blick auf spezielle Veranstaltungen in mehreren Exemplaren, aber auch von Studierenden und Schülern gekauft werden - wenn auch nicht so intensiv, wie es bei den angelsächsischen, auf spezielle Lehrveranstaltungen bezogenen Textbüchern der Fall ist.

Mit der Öffnung des Internets auch für die Wissenschaftsverlage änderte sich diese sozusagen friedliche Situation. Produkte und Dienstleistungen, die auf Bildung und Wissenschaft abzielen, versprechen in der globalen Perspektive große Gewinne. Ein hoch-profitabler Wissenschaftsmarkt entwickelte sich vor allem mit Blick auf die internationalen Zeitschriftenmärkte. Der Wissenschaftsmarkt ist big business geworden, mit den zu erwartenden Konsequenzen der Monopolisierung,

552 Das andere große Segment des Wissenschaftsmarktes sind die seit den 60er Jahre entwickelten Online-Datenbanken, die zunächst nur als Referenz-Datenbanken weltweit aufgebaut und angeboten wurden (bibliographische Information, eventuell angereichert durch Abstracts und Keywords/Deskriptoren), die dann aber weitgehend durch Volltext-Datenbanken ersetzt wurden. Dazu gehören in ständig wachsendem Umfang (nicht textorientierte) Daten/Fakten-Datenbanken. 553 Nach (STM 2018), 22 werden weltweit von Bibliotheken 8 Mrd für akademischen und wissenschaftlichen Inhalt ausgegeben. Akademische Bibliotheken erbrachten für Verlage traditionell die primären Erlöse aus dem Zeitschriftenmarkt, geschätzt zwischen 68 und $75 \%$ des gesamten Erlös aus Zeitschriften. Andere Erlöse durch „corporate subscriptions (15-17\%), Werbung 4\%, etc. 
vor allem auf den Zeitschriftenmärkten, besonders bei den Zeitschriften für die MINT/STM-Fächern. Im Grunde dominieren hier nur fünf große Verlagskonsortien diesen Markt: Elsevier, Springer Nature, Wiley, Taylor \& Francis und Sage. Monopole ruinieren für die Nutzer das Preis-/Leistungsverhältnis. Für die Informationsversorgung der Wissenschaft durch Bibliotheken wurden die ansteigenden Kosten, insbesondere durch die Subskriptionskosten für die Zeitschriften, zu einem Problem. In der Öffentlichkeit wurde es als zunehmend unangemessen oder sogar empörend angesehen, dass die großen Verlagskonsortien mit Gewinnen von ca. $30 \%$ kalkulieren konnten und für die Nutzungserlaubnisse der in der Wissenschaft erstellten, öffentlich finanzierten Wissensobjekte i. d. R. nichts bezahlen mussten. Auf dieses Geschenkmodell wird näher in 14.5 eingegangen, zunächst in 14.3 auf die Folgen der Monopole bzw. der Oligopole und danach auf die aktuell noch geltende Marktsituation - in 14.4 auf den Wissenschaftsmarkt allgemein und in 14.4.2 speziell auf den für Zeitschriften.

\subsection{Zu den Monopolen auf den Wissenschaftsmärkten}

\subsubsection{Hochpreispolitik}

Monopole setzen Wettbewerb auf den Märkten aus. Monopole erlauben es, eine Hochpreispolitik zu verfolgen. Dass es zumindest fünf große Verlagkonsortien für die Zeitschriftenpublikation gibt, relativiert den Monopolcharakter nicht, auch Oligopole erlauben keine wirkliche Konkurrenz. Produkte in der Wissenschaft haben für sich schon Monopol-/Einzigartigkeitscharakter. Das gilt für den einzelnen Artikel, der, wenn er verlangt wird und dieser nicht in der Bibliothek verfügbar ist, nicht einfach gegen einen anderen ausgetauscht werden kann. Aber dieses Monopol gilt auch, wenn auch etwas weniger stark, für die Zeitschriften selbst. Die Hochpreispolitik bezieht sich zudem nicht nur auf eine einzelne Zeitschrift. Die wenigen großen Zeitschriftenverlage sind immer mehr dazu übergegangen, die Lizenzangebote in großen Bündeln zusammenzufassen. Dadurch werden die extrem nachgefragten und sehr teuren Zeitschriften mit anderen, viel weniger nachgefragten zusammengepackt und gemeinsam lizenziert. Damit diktieren die Verlage die Preispolitik über die gesamten Bündel. Die dadurch entstehenden hohen Kosten sind immer weniger in Einklang zu bringen mit den Budgets der meisten Bibliotheken. Hier ein Beispiel für die Kosten der vermutlich eher überdurchschnittlich gut finanziell ausgestatteten Bibliothek der ETH Zürich, welches (Gutknecht 2015) 554 entnommen ist:

554 (Gutknecht 2015) Zahlungen der ETH Zürich an Elsevier, Springer und Wiley nun öffentlich. 
Alleine die Ausgaben für Zeitschriften, E-Books und Datenbanken an die drei Verlage Elsevier, Wiley und Springer Nature betragen zusammen 7.78 Mio CHF und machen somit fast die Hälfte des gesamten Erwerbungsbudget der ETH-Bibliothek (17.751 Mio CHF) aus.

Die effektiven Ausgaben an die klassischen Subskriptionsverlage Elsevier und Wiley sind in den letzten Jahren weiter massiv gewachsen - mehr Open Access möchte man, mehr Closed Access bezahlt man.

Mit 3.55 Mio CHF Ausgaben für Elsevier Zeitschriften im Jahre 2014, toppt die ETH alle britischen Universitäten. Das University College London gab 2014 den größten Betrag von ca. 2.5 Mio CHF (£1’657’434) für Elsevier Zeitschriften aus. Oxford sogar „nur“ ca. 1.5 Mio CHF (£990’774).

Auch im Vergleich zu amerikanischen Universitäten liegen die Ausgaben der ETH an Elsevier im Spitzenbereich. Cornell bezahlte im Jahr 2013 ca. 2.4 Mio (USD 2'468'244). Das MIT beziffert in seinem Elsevier Factsheet seine Ausgaben auf über 2 Mio USD, wobei das MIT - wie in den USA üblich - nicht alle Journals von Elsevier abonniert hat.

Gemäß ETH-Jahresbericht 2014 sind an der ETH gut 7000 Personalstellen mit Lehre und Forschung beschäftigt. D. h. pro wissenschaftlicher Stelle kostet der Elsevier Zugang gute 500 CHF. Natürlich verflacht sich diese Zahl wenn man noch die 18616 Studierenden dazuzählt, aber das ist ja bekanntlich nicht die Hauptnutzungsgruppe von Zeitschriften.

Dass die finanziellen Mittel von Bibliotheken wie der für die ETH für Kauf oder Lizenz von Publikationsprodukten überwiegend an die proprietären Produkte der großen Verlage gehen, muss nicht so sein. Es gibt schon jetzt in der Form von Open-Access-Produkten realistische Alternative zu den proprietären Produkten (vgl. 14.7). Dies belegt Gutknecht mit dem Hinweis, dass für die ETH, vermutlich aber auch für Bibliotheken allgemein, angesichts der hohen Subskriptionskosten ein Wechsel auf die Finanzierung von Open-Access-Produkten über das APC-Modell finanziell machbar wäre. ${ }^{55} \mathrm{Ob}$ sich die Kosten dadurch sogar reduzieren würden, ist in kurzfristiger Perspektive nicht zu erwarten. ${ }^{556}$ Aber ein gewisser Nachhaltigkeits-

555 Diese Einschätzung, dass APC-Gebühren durch die Bibliotheken erbracht werden könnten, wird durch die Studie von Nina Schönfelder bestätigt (Schönfelder 2019): Eine Untersuchung bei fünf Universitären und einer Forschungseinrichtung hat ergeben, dass für die aus der Grundfinanzierung entstandenen Werke die APC-Gebühren erbracht werden können. Dies wird bei den drittmittelfinanzierten Werken aber nur dann möglich sein, wenn die entsprechenden Förderorganisation dafür einspringen. Problematisch könnte die ABC-Finanzierung sein vor allem für publikationsintensive Hochschulen bzw. außeruniversitäre Forschungseinrichtungen. Ein ähnliches Problem zeichnet sich auch bei dem DEAL-Modell ab (vgl. 14); Schönfelder schlägt einige Modelle vor, wie diese Gebühren „gestemmt“ werden können. Sie reichen von „Diamond Open Access“ bis hin zu Crowdfunding-Modellen. Durch die DEAL-Vereinbarung zeichnet sich allerdings ab, dass APC durch PAR (publish\&read) abgelöst werden könnte. Bislang (Ende 2019) haben sich allerdings erst Wiley und Springer Nature dieser Vereinbarung angeschlossen.

556 Zu den Kosten insgesamt vgl. die Angaben von (Pflüger 2016, 487): „Die öffentlichen Ausgaben für die wissenschaftlichen Bibliotheken stiegen binnen zehn Jahren um $40 \%$ von 220 Mio EUR in 2003 auf 311 Mio EUR in 2013 und dürften heute bei 330 Mio EUR liegen. Davon entfallen inzwischen 
effekt ist nicht zu verkennen. Zum einen wären alle APC finanzierten Publikationen (wenn sie tatsächlich dem Open-Access-Standard entsprechen) nicht nur für die ETH-Mitglieder, sondern sofort weltweit frei für jedermann zugänglich. Zum anderen erhöht sich sukzessive der Anteil der Open-Access-Publikationen mit der Folge der Reduzierung der Abhängigkeit von den proprietären Publikationen. Das ist z. B. auch das Modell und die Erwartung von Einrichtungen wie der MPG in Deutschland: schrittweise Transformation in Richtung Open Access-Informationsmärkte durch Umschichtung (nicht unbedingt Einsparen) der aufzubringenden Bibliothekskosten von Subskriptionsgebühren in Open-Access-Kosten. Das wird aktuell bestätigt durch Vereinbarungen im Rahmen des DEAL-Projekt in Deutschland, bei dem die bisherige APC-Gebühr durch eine am PAR (publish\&read) orientierte Gebühr abgelöst wird (ausführlich dazu in 14.8).

\subsubsection{Monopole über Impact Factor?}

Das langanhaltende Monopol der Verlage beruhte nicht nur auf dem technischen, organisatorischen und editorischen Knowhow. Monopole sind auch durch einen qualitativen Faktor entstanden, der ursprünglich lediglich zur Orientierung für die Wissenschaft gedacht war. ${ }^{557}$ Damit ist der schon angesprochene Impact Factor gemeint, der angibt, wie häufig Artikel in einer Zeitschrift von Autoren anderer wissenschaftlicher Zeitschriften zitiert werden. Der IF gibt nur den Durchschnittswert für die Zitierungen aller Artikel in dieser Zeitschrift an, ist also keineswegs ein Wert für die Zitierhäufigkeit eines bestimmten Artikels. Viele Artikel selbst in einer Zeitschrift wie Nature mit hohem IF werden überhaupt nicht zitiert. Der IF ist somit eher eine Bewertung der Zeitschrift. Zeitschriften, so (Ziegler 2019), ${ }^{558}$

120 Mio EUR auf die Lizenzierung digitaler Medien, deren Anteil bei den Universitätsbibliotheken jetzt $60 \%$ beträgt. “ Die Lizenzierung bezieht sich im Wesentlichen auf die wissenschaftlichen Zeitschriften.

557 Der Impact Factor (IF) von Zeitschriften wurde zuerst in den 1960er Jahren vom Institute for Scientific Information (ISI) entwickelt. Nach verschiedenen Übernahmen durch Verlagskonsortien wird heute der IF von Clarivate Analytics verwaltet - sowohl für die sozialwissenschaftlichen Fächer im Social Sciences Citation Index als auch für die MINT-Fächer im Science Citation Index. Jährlich gibt Clarivate Analytics Journal Citation Reports (JCR) heraus. Hier einige Daten aus dem Journal Citation Reports (JCR) von Clarivate Analytics von Juni 2019 - https://bit.ly/2ZfmMvj. The report covered 11,877 journals from across 81 countries.

283 new journal titles were added to the report, 138 of which are fully open access. The JIF of $64 \%$ of the journals covered in the report increased.

The aggregate JIF increased in $90 \%$ of the 236 research categories.

17 journals were restricted from the report after investigations revealed abnormal citation behavior. 558 (Ziegler 2019) Die Bedeutung der Verlage ändert sich. 
sind „Renommee-Maschinen“ geworden und „machen Karriere“. Die Berufung auf eine Professur wird häufig, vor allem in STM-Fächern, in hohem Maße von Publikationen in Zeitschriften mit hohem IF abhängig gemacht, auch wenn Arbeiten der betreffenden Person tatsächlich nur gering von der Fachwelt zitierend wahrgenommen wurden. ${ }^{559}$ Ebenso orientiert sich zuweilen die Einschätzung der Qualität einer gesamten Hochschule am IF. Aus all dem folgt, dass der IF ein hervorragendes Mittel für Verlage ist, Wissenschaftler quasi dazu zwingen, in deren Zeitschriften zu publizieren. Vor allem jüngere Wissenschaftler sind für ihre Karriere darauf angewiesen, in Zeitschriften mit hohem IF zu publizieren. Eine etablierte Hoch-IF-Zeitschrift hat praktisch ein Monopol, das durch eine vom Gegenstandsbereich ähnlich ausgerichtete Zeitschrift nicht ersetzt werden kann.

Verlage tun so als ob sie für die Qualität der in einen Artikel eingeflossenen wissenschaftlichen Arbeit verantwortlich wären. ${ }^{560}$ Verlage stellen aber überwiegend nur die Plattformen und ihr spezielles Knowhow bereit. Für die Qualität der Inhalte sorgt die Wissenschaft selbst, traditionell über das Peer Reviewing, also für die Bewertung der wissenschaftlichen Qualität durch ausgewiesene Mitglieder der jeweiligen Community. Auch die Rekrutierung von qualifizierten Autoren erfolgt nicht von den Verlagen, sondern von den Wissenschaftlern in den Herausgebergremien. Verlage haben es verstanden, das Renommee, dass ursprünglich alleine durch die Verantwortung der Learned societies für die Qualitätssicherung der Veröffentlichungen entstanden war, auf sich zu übertragen und sich somit unersetzlich zu machen. Allerdings - und auch das gehört zu der in diesem Kapitel angesprochenen Transformation - wird der IF zunehmend zugunsten von alternativen Messverfahren in Frage gestellt - nicht nur, weil durch den IF die tatsächliche Nutzung eines Artikels gar nicht gemessen wird, sondern auch, weil der IF als ein von der kommerziellen Wirtschaft organisiertes und auch kontrolliertes Messverfahren recht deutlich deren Interessen unterstützt. Als Alternative bzw. Ergänzung zum IF wird auch der sogenannte Hirschfaktor (auch h-Faktor genannt) verwendet. Er misst anders als der IF nicht die Rezeption einer gesamten Zeitschrift, sondern bezieht sich auf

559 Der IF mit dem Anspruch auf Qualitätsnachweis wird durchaus auch in der Politik kritisch gesehen. Der EU-Rat (Council 2016, vgl. FN 626) empfiehlt eine stärkere wirkungsbezogene Bewertung: ,assessing scientific quality should be based on the work itself and be broadened to include an assessment of the impact of science on society at large“. Entsprechende Initiativen für eine bessere Qualitätssicherung in den „review and evaluations systems“sollten gefördert werden; ebenso Anreizsysteme geschaffen werden, „to reward researchers (and research stakeholders) for sharing the results of their research for reuse“ (ebda.). Ebenso sollten offene Publikations- und Nutzungspraktiken bei Projekten und Berufungen angemessen anerkannt und belohnt werden. 560 Kritik an der Leistung von Verlagen (am Beispiel der Edition eines Sammelbands) von (Lorenz M. Hilty 2015) Was leisten Wissenschaftsverlage heute eigentlich noch? netzpolitik.org 26.08.2015 https://bit.ly/3fu1xsK 
die Rezeption der Arbeiten eines einzelnen Wissenschaftlers durch andere Wissenschaftler. Er gibt also an, inwieweit eine gewisse Anzahl von Publikationen eines Wissenschaftlers häufig in Veröffentlichungen anderer Wissenschaftler zitiert wird.

Alternativ zum IF ist auch der von Elsevier entwickelte und propagierte CiteScore-Index. CiteScore misst die Häufigkeit der Zitierung eines Artikels in einer dreijährigen Periode. ${ }^{561}$ CiteScore ist ein Bestandteil eines umfassenden "basket of metrics", wodurch, neben allgemeinen Altmetrics-Formen, auch Faktoren wie z. B. das Ausmaß der Zusammenarbeit (collaboration) mehrerer „Autoren“ an einem Artikel, die Finanzierung durch Förderorganisationen, die kommerzielle Nutzung der Arbeit, die soziale Wirkung eines Artikels, auch die Qualität des Peer Reviewing allgemein berücksichtigt werden. ${ }^{562}$ Das könnte sich zu einer Konkurrenz zum IF entwickeln, der sich ja nicht auf einzelne Artikel, sondern auf die Zeitschrift insgesamt bezieht. Generell kommen immer mehr neue, vom Zitierungsverhalten unabhängige alternative Messverfahren zum Einsatz. Mit diesen Altmetrics-Formen ${ }^{563}$ kann das tatsächliche Nutzungsverhalten gemessen, z. B. über Anzahl der Downloads, die Aufmerksamkeit und Verbreitung, die ein Beitrag in den unterschiedlichen Medien (darunter auch soziale Medien wie Blogs, News oder Twitter oder auch Publikationsserver/Repositorien) erfahren hat, aber auch Faktoren, inwieweit ein Beitrag auch Eingang in öffentliche, mediale oder auch politische Diskussion und Entscheidungsprozesse gefunden hat. In gewisser Hinsicht wird damit auch das qualitative Peer Reviewing durch quantitative Messen der Rezeption in verschiedenen Medien ersetzt. Solche Messzahlen sind vor allem durch die elektronischen Umgebungen heute leichter zu ermitteln. Als Vorteil wird auch angesehen, dass die Abhängigkeit der Qualitätsmessung vom Urteil der (i. d. R. etablierten und bestehenden Paradigmen verpflichteten) Peer Reviewers reduziert und damit die Durchsetzung neuer Entwicklungen in der Wissenschaft erleichtert wird (vgl. dazu $14.11 \mathrm{zu}$ Research Intelligence). In der wissenschaftlichen Praxis erweisen sich allerdings weiterhin der IF, zunehmend auch der CiteScore, als stabile und weitgehend akzeptierte Indikatoren zur Qualitätssicherung von wis-

561 CiteScore: a new metric to help you track journal performance and make decisions, 8.12.2016: "CiteScore metrics calculate the citations from all documents in year one to all documents published in the prior three years for a title. This offers a more robust and accurate indication of a journal's impact. As an example, to calculate a 2015 value, CiteScore counts the citations received in 2015 to documents published in 2012, 2013 or 2014. This number is divided by the number of documents indexed on Scopus published in 2012, 2013 and 2014”. - https://bit.ly/2hmSC2O.

562 Vgl. (Colledge/James 2015) (beide 2015 tätig bei Elsevier): A "basket of metrics"-the best support for understanding journal merit. Kritisch zu CiteScore und dem Umfeld im "basket”: (Straumsheim 2016) How to measure impact.

563 Altmetrics: What are altmetric? Capturing the online attention surrounding scholarly content https://bit.ly/1Vxq6IR. 
senschaftlichen Arbeiten. Das gilt auch für (bislang überwiegend auf Zeitschriften bezogene) Open-Access-Produkte (vgl. aber 14.11). Allerdings sieht die DFG (im September 2018) durchaus die Notwendigkeit, Maße wie den IF auch kritisch für Open-Access-Publikationen zu überprüfen:

Der „Wandel hin zu Open Access“ setze auch einen grundlegenden Umbau der Systeme zur Leistungsmessung durch Forschungsorganisationen voraus. Insbesondere ist eine Abkehr von Indikatoren wie dem Journal Impact Factor notwendig. Hier ist noch ein weiter Weg zu gehen. ${ }^{564}$

Beide Faktoren, IF und CiteScore, sind im hohen Maße abhängig von den Interessen der sie betreibenden kommerziellen Organisationen. Sie verstärken die Bindung oder sogar Abhängigkeit der Wissenschaftler an Produkten dieser Organisationen. Das umfassende Angebot von Elsevier im "basket”, das sicherlich nicht nur als Informationsmaterial gedacht ist, ist ein Hinweis darauf, dass die großen Verlagskonsortien sich zwar nicht vom Geschäft des Publizierens abwenden, aber doch neue Geschäftsmodelle im Umfeld von Wissenschaft entwickeln (vgl. $14.11 \mathrm{zu}$ den Perspektiven kommerzieller Verlage).

\subsection{Zu den kommerziellen proprietären Informationsmärkten}

Voraussetzung für das Entstehen kommerzieller proprietärer Informationsmärkte ist, dass die entsprechenden kommerziellen Akteure, Unternehmen wie Verlage, sich die Erlaubnis zur kommerziellen Nutzung von den Urhebern unter vereinbarten Bedingungen einholen. Verlage produzieren kein Wissen, sie produzieren auch keine Wissensobjekte. Das machen die Urheber selbst, aber Verlage (allgemein: Content Provider) setzen diese Objekte in handelbare Informationsobjekte auf den Märkten um. Für den Erwerb der Nutzungsrechte an den Wissensobjekten wird i.d.R. von den Verlagen nichts an die Autoren bzw. an deren Institutionen bezahlt - das gilt vor allem für die die Informationsmärkte dominierenden Zeitschriftenartikel.

\subsubsection{Allgemeine Daten zu den Informationsmärkten}

Quantitative Aussagen zu den auf Erlös abzielenden Informationsmärkten werden jedes Jahr von der WIPO über die World Intellectual Property Indicators (WIPO)

564 Stellungnahme der DFG zur Gründung von „cOAlition S“ zur Unterstützung von Open Access vom 4.9.2018 - https://bit.ly/2Humea5. Vgl. dazu der EU Council 2016 (FN 624). 
vorgelegt, aktuell (WIPO 2018) und von STM (International Association of Scientific, Technical and Medical Publishers), aktuell (STM 2018). ${ }^{565}$ Die WIPO-Daten tragen der weltweiten Entwicklung Rechnung, während die von STM vorgelegten Berichte zum Umfang der kommerziellen Informationsmärkte sich in erster Linie auf die englischsprachigen Publikationen und Aktivitäten beziehen - mit Schwerpunkt auf die Zeitschriftenmärkte (vgl. 14.4.2). Die Daten der WIPO sind allgemein gehalten, während die von STM mehr ins Detail gehen - allerdings mit dem Nachteil der weitgehenden Beschränkung auf den englischsprachigen Bereich (s. unten).

In der Abteilung Creative Economy von WIPI/WIPO werden in der Zusammenarbeit der International Publisher Association (IPA) und der WIPO Daten zu den drei Marktsegmenten ,trade; educational; and STM publishing“ zusammengetragen. ${ }^{566}$ Die Daten von (WIPO 2018) zeigen deutlicher als die von STM den immer größer werdenden Anteil asiatischer Länder wie Indien und Japan, aber vor allem von China, an den Informationsmärkten in den erwähnten drei Segmenten. Gesamtdaten für alle drei Bereiche konnten von der WIPO für 11 Länder erhoben werden. Deren Gesamterlös betrug 2017 \$248 Mrd, davon alleine 202,4 Mrd für China, gefolgt von \$25,9 Mrd für USA und an dritter Stelle Deutschland mit 5,8 Mrd. China produzierte 2017 über die drei Sektoren hinweg 65 Mio Titel. Die Einnahmen im „Educational Sector“ betrugen 2017 für China 72,8 Mrd, gefolgt von USA mit 7.8 Mrd.

\subsubsection{Zeitschriftenmarkt der Informationswirtschaft}

Der Zeitschriftenmarkt ist auch heute noch der größte und lukrativste für die kommerziellen Informationsmärkte - aber auch der am schnellsten wachsende Open-Access-Markt (vgl. 14.7). Zeitschriftenartikel sind nach wie vor das wichtigste Medium für den wissenschaftlichen Informationsaustausch. Zeitschriften sind erst relativ spät (im 17. Jahrhundert) als indirekte Form der Kommunikation in der

565 "STM is the leading global trade association for academic and professional publishers. It has over 150 members in 21 countries who each year collectively publish over $66 \%$ of all journal articles and tens of thousands of monographs and reference works. STM members include learned societies, university presses, private companies, new starts and established players." (STM 2018) An overview of scientific and scholarly publishing."

566 Eine weltweite Erhebung ist schwierig, da in den meisten Ländern keine Instanz alleine dafür zuständig ist. Lediglich in Kanada (Statistics Canada), Japan (Japan Copyright Office) und China (National Copyright Administration of China) werden die statistischen Daten von einer Regierungsagentur erhoben. Bei den anderen Ländern sind die wichtigsten Instanzen für die Daten die National Publishers' Association (NPA). Allerdings sind in den wenigstens Ländern alle Verleger Mitglieder der jeweiligen NPA. Dazu kommen erhebliche methodische Unterschiede bei der Erhebung und Einordnung der Daten. 
Wissenschaft entwickelt worden. Aber schon immer seit Wissenschaft betrieben wurde, haben sich Formen entwickelt, durch die sich Wissenschaftler untereinander austauschen (vgl. (Parthey/Umstätter 2002). Bis zur Entwicklung des Buchdrucks mit bewegten Lettern waren das eher direkte Kommunikationsformen unter sehr begrenzten Teilnehmern, z. B. Gespräche, Briefwechsel, Kopieren oder Ausleihen von Handschriften. ${ }^{567}$

Durch die Möglichkeit des Druckens, der Vervielfältigung und Verbreitung wurden indirekte, medial unterstützte Kommunikationsmöglichkeiten möglich, die auch dafür verantwortlich waren, dass seit dem 16., 17. Jahrhundert immer mehr Personen wissenschaftlich tätig waren und entsprechenden Output produzierten, der anderen Wissenschaftlern zugänglich gemacht werden sollte. Das ist der Beginn der formalisierten indirekten Kommunikation über Zeitschriften. Diese etablierten sich Mitte des 17. Jahrhunderts und wurden getragen von wissenschaftlichen Akademien und wissenschaftlichen Gesellschaften/Fachgesellschaften (learned societies). ${ }^{568}$ Hier gab es offenbar keine gravierenden Konflikte zwischen der kommerziellen Verwertung und dem Anspruch der Mitglieder dieser Organisationen auf freie Nutzbarkeit der in den Zeitschriften publizierten Materialien. Fachgesellschaften sind auch heute noch produktive Akteure auf den Publikationsmärkten, ${ }^{569}$ die das Publizieren nicht nur durch Mitgliedsbeiträge finanzieren, sondern auch selbst

567 Ein Überbleibsel dieser direkten Kommunikation war lange Zeit der Austausch von Sonderdrucken, welche die Verlage vor allem für kleinere Arbeiten wie Artikel in Zeitschriften oder Sammelbänden in überschaubarer Anzahl bereitstellten. Die Verteilung an Kollegen mag in vielen Fällen eher der Reputationssteigerung gedient haben als der ausführlichen Lektüre. Aber es war (und das wird teilweise auch heute noch von manchen Verlagen bedient und von Wissenschaftlern genutzt) doch ein wichtiges Kommunikationsmittel, um zumindest zu erfahren, woran Andere derzeit arbeiten. Direkte Kommunikation erfährt in Zeiten des Internets eine spezielle Renaissance. Mit geringem Aufwand kann elektronisch direkter Kontakt zu dem Autor eines Artikels, den man gerade benötigt, hergestellt werden, mit der Bitte, doch diesen Artikel als Kopie zu schicken. Das funktioniert i. d. R. umgehend, auch zu Autoren, mit denen man bislang keinen Kontakt hatte. Das ist auch legal, solange die Kopien für den persönlichen Gebrauch genutzt werden. Ganz so sicher kann man sich allerdings nicht mehr sein; denn aus der Regelung für die Privatkopien in $\S 53$ UrhG ist Abs. 2, 1 weggefallen: „Vervielfältigungen zum privaten und sonstigen eigenen Gebrauch”; „zum eigenen wissenschaftlichen Gebrauch, wenn und soweit die Vervielfältigung zu diesem Zweck geboten ist und sie keinen gewerblichen Zwecken dient“.

568 (Kant 2002) Disziplinäre Gesellschaften als Träger von Fachzeitschriften. Fast zeitgleich erschienen 1665 Journal des Sçavans in Paris und die Philosophical Transactions in der Londoner Royal Society. Vgl. (Zott 2002): Der Brief und das Blatt.

569 Z. B. Physik Journal, die Mitgliederzeitschrift/das Mitteilungsorgan der Deutschen Physikalischen Gesellschaft (DPG) mit einer Auflage von über 60.000 Exemplaren, ist die bedeutendste Physikzeitschrift im deutschsprachigen Raum - nicht im Handel erhältlich. Die Publikation geschieht in Zusammenarbeit von DPG mit dem Verlag Wiley-VCH. 
kommerzielle Akteure auf den Publikumsmärkten sind und dadurch auch ihre Organisationen mitfinanzieren. ${ }^{570}$ Zur finanziellen Absicherung, aber auch um das Publizieren aus der Wissenschaft selbst heraus abzusichern, haben sich verschiedentlich Fachgesellschaften zu Konsortien zusammengeschlossen. ${ }^{571}$ Fachgesellschaften kooperieren oft für die Publikation und Verbreitung mit kommerziellen Verlagen. ${ }^{572}$ Hier einigen Daten zur Gegenwart des Zeitschriftenmarkts:

Die fünf größten Verlage sind mit Blick auf Zeitschriften (Z): Springer Nature $(>3.000 \mathrm{Z}$ ), Elsevier (2.500 Z), Taylor \& Francis (2.500 Z), Wiley (1.700 Z) und Sage (>1000 Z) (nach STM 2018). In der STM-Industrie sind, nach (STM 2018), ca. 110.000 Menschen beschäftigt (davon 40 \% in Europa) - dazu kommen 20-30.000 Personen, die indirekt der STM-Produktion zuarbeiten. (STM 2018) geht von 33.100 aktiven peer-reviewed englischsprachigen Zeitschriften (Mitte 2018) aus. Nach Ulrich's Web Directory steigt die Anzahl der wissenschaftlichen Zeitschriften auf 42.491, wenn man die nicht englischsprachigen Zeitschriften dazu zählt (zit. nach FN 24 aus STM 2018, 41). Davon sind nach (STM 2018) weltweit ca. 23.000 kommerzielle wissenschaftliche Zeitschriften. Ca. 10.000 Zeitschriften-Verlage sind dafür zuständig. Von diesen sind 5.000 in der SCOPUS-Datenbank aufgenommen.

So gut wie alle Verlage bieten den Online-Zugriff auf die Zeitschriftenartikel an. 477 Verlage mit ca. 2.334 Zeitschriften operieren auf Not-for-profit-Basis. Zusammen werden über diese Zeitschriften mehr als 3 Millionen Artikel pro Jahr produziert und veröffentlicht. Der Zuwachs pro Jahr ist inzwischen auf etwa $5 \%$ bei Zeitschriften und $4 \%$ bei Zeitschriftenartikeln angestiegen. Der Grund für den Anstieg liegt in erster Linie darin, dass es immer mehr aktive Wissenschaftler gibt. Je nach Erhebung geht man von 7-8 Millionen aus. Davon sind $80 \%$ Wissenschaftler, die bislang nur einen Artikel produziert haben. China hat inzwischen mit $19 \%$ den größten Anteil an produzierten Artikeln, gefolgt von den USA mit $18 \%$, Indien mit $5 \%$ und Deutschland, England und Frankreich mit jeweils 3-4\%. Elsevier's SCOPUS deckt 22.000 peer-reviewed Zeitschriften mit 69 Mio Artikeln (Zuwachsrate 3 Mio/Jahr) von 5.000

570 (STM 2018) berichten, dass alleine im UK 279 Learned societies Zeitschriften, überwiegend aus dem STM-Bereich, publizieren. Auch in Deutschland sind Fachverbände weiter Akteure auf den Publikationsmärkten. So ist die Gesellschaft Deutscher Chemiker (GDC) Eigentümer bzw. Miteigentümer von wichtigen chemischen Fachzeitschriften, auch in Zusammenarbeit mit anderen europäischen Fachgesellschaften unter dem Dach von ChemPubSoc Europe - https://bit.ly/2lKqeej. Die GDC bzw. auch andere Gesellschaften unter dem Dach von ChemPubSoc arbeiten zusammen mit Wiley-VCH Gmbh \& Co.KgaA.

571 (STM 2018, 44): „Recently, a group of prestigious not-for-profit scientific membership societies launched the Scientific Society Publisher Alliance (SSPA), 45 an initiative focused on building awareness of and support for publication of scientific research by scientist-run scientific societies. The SSPA seeks to emphasize the value of publishing vital scientific research in scholarly journals managed, edited and peer-reviewed by working scientists."

572 Wiley ist hier mit mehr als 700 Kooperationen führend; aber auch andere wie Taylor \& Francis bedienen dieses Marktsegment (STM 2018, 44). 
Verlagen ab. 1science 1 findr listet ${ }^{573} 67.000$ akademische/wissenschaftliche Objekte und 90 Mio peer-reviewed Artikel. Digital Science's Dimensions indexiert 90 Mio wissenschaftliche Dokumente - davon sind $85 \%$ Zeitschriftenartikel. Informa's wizdom.ai umfasst 73.000 Zeitschriften mit 90 Mio Publikationen (alles nach STM 2018, Abschnitt 2.5).

Leicht andere Daten für den Umfang des Zeitschriftenmarktes, ebenfalls aus (STM 2018): Die CrossRef-Datenbank weist 97 Mio DOIs nach, von denen 73 Mio Artikel aus Zeitschriften sind. Google Scholar hatte schon 2014 zwischen 100 und 150 Mio Dokumente angezeigt, wovon ein großer, wenn nicht der größte Teil vermutlich auch solche aus der Wissenschaft sind. Web of Science weist in der „Core Collection“ im Juni 201970 Mio Artikel nach - eine Teilmenge der 150 Mio Objekte in der WoS. Eine besonders gewichtige Teilmenge wissenschaftlicher Zeitschriften bzw. deren Artikel sind die 11.655 Zeitschriften, die in Alaivate Analytics (2018) Journal Citation Reports zusammengefasst sind und die zusammen 2,2 Mio Objekte nachweisen. Dies sind die am meisten zitierten wissenschaftlichen Zeitschriften.

Der gesamte Erlös auf dem STM-Markt (Zeitschriften, Bücher, Datenbanken etc.) betrug nach (STM 2018) 2017 \$ 25,7 MRD - der Anteil von Wissenschaft/Technik \$ 13,8 MRD (davon ca. \$ 10 MRD aus englischsprachigen STM Zeitschriften) und der von Medizin \$11,9 Mrd. Ca. 41\% der globalen STM Gesamteinahmen werden in den USA erzielt, $25 \%$ von Europa und Mittelost, $26 \%$ von Asien/ und $6 \%$ vom Rest der Welt. Die Kosten für Forschung und die Kommunikation der Forschungsergebnisse, die in Fachzeitschriften erscheinen, wurden (nach STM 2018, 24) für 2008 auf $€ 175$ MRD geschätzt davon $€ 116$ MRD alleine für die Forschung, ${ }^{574} € 25$ für die Publikation (Publizieren und Bibliothekskosten) und £34 für das Lesen/Nutzen. Das Publizieren alleine kostet ca. £4.9 MRD; das entspricht knapp $3 \%$ der Gesamtkosten.

In den letzten Jahren verstärkt sich auch das Retail-Geschäft für Zeitschriftenartikel. Dieser direkte Online-Zugriff zu einzelnen Zeitschriftenartikel wird durch komfortable kostenpflichtige Dienstleistungen der Verlage wie ScienceDirect ${ }^{575}$ ermöglicht, i. d. R. über einen von der Bibliothek eingerichteten Account. Möglich ist die Nutzung auch direkt mit einem persönlichen Zugriff. Ein solcher Zugriff wird aber weltweit in erster Linie von bzw. aus gut ausgestatteten Hochschulen oder For-

573 1science 1findr - https://bit.ly/36Z7gU1. 1science wurde von Eric Archambault entwickelt und wurde später von Elsevier übernommen.

574 Diese Angabe ist tatsächlich eher als geschätzt denn als verifiziert anzusehen, nicht zuletzt auch deshalb, weil kaum auszumachen ist, welche Forschung explizit in Zeitschriftenartikeln veröffentlicht wird. Auch die quantitative Angabe von £116 MRD ist viel zu klein angesetzt. Allein in Deutschland wurde für Forschung 2014 ca. Euro 84 Mrd ausgegeben (davon 2/3 von der Privatindustrie. Damit liegt Deutschland weltweit an vierter Stelle nach USA, China und Japan (forschungsportal.net: So viel Geld für Forschung wie nie zuvor, 31.3.2017 - https://bit.ly/ 2lDsN1U). Detaillierter im Wikipedia-Artikel: Liste der Länder nach Ausgaben für Forschung und Entwicklung - https://bit.ly/2lCsch4; darin auch Verweis auf Daten von Visual Capitalist: The Global Leaders in R\&D Spending, by Country and Company, 13.10.2017 - https://bit.ly/2k5GjuJ.

575 ScienceDirect - https://bit.ly/3608pJC. 
schungseinrichtungen wahrgenommen. In ihnen arbeitet aber nur ein Teil der ca. 60 Millionen Menschen, die als weltweit Forschende, Lehrende und Lernende tätig sind. Dazu kommen die vielen in privaten Unternehmen, öffentlichen Verwaltungsorganisation oder auch privat Forschende (vermutlich noch einmal mindestens 60 Millionen) sowie die Studierenden ${ }^{576}$ (zu den Open-Access-Zeitschriftenmärkten vgl. 14.7).

\subsubsection{Bücher auf den Informationsmärkten}

Buchproduktion ist auch heute noch für Bildung und Wissenschaft ein wichtiger Bestandteil kommerzieller Informationsmärkte. Nach (STM 2018) wurde 2017 über den STM Buchmarkt weltweit etwa ca. 3,3 Mrd an Erlös erzielt. Davon entfallen \$ 719 Mio auf wissenschaftliche und technische Bücher und 2,48 Mrd auf Medizinbücher. Der Anteil der gedruckten Bücher am Gesamtmarkt geht um ca. 4\%/Jahr zurück, während die Wachstumsrate für E-Books 4-6\% pro Jahr beträgt. E-Books haben auch einen zunehmend größeren Anteil an den Bibliotheksbudgets. Anders als bei Zeitschriften werden Fachbücher intensiver von Endnutzern erworben; aber insgesamt bleiben auch hier die Bibliotheken die wichtigsten Käufer/Lizenznehmer.

Im Vergleich zu den internationalen Daten hier einige Daten aus Deutschland, die über den Börsenverein des Deutschen Buchhandels bereitgestellt werden: ${ }^{577}$ Der hiesige Buchmarkt besteht im Wesentlichen aus zwei Teilen, relativ stabil in den letzten 3 Jahren zu 2/3 aus den nicht wissenschaftlichen Bereichen (Belletristik, Kinder, Reisen, Ratgeber) und 1/3 aus den Wissenschaften, einschließlich Schule und Sachbuch. Einige Daten zur Buchproduktion 2018 (gesamt 71.548), z. B. Information, InfWiss: 1.500 (davon Informatik 650); NaturWiss und Mathematik:

576 (Dinleavy 2018) Open Access knowledge. Digital style guide.

577 In Tabellenform durch Börsenverein 2018: https://bit.ly/3aivz1i. Im Verlagsbuchhandel waren 2018 ca. 25.000 Personen beschäftigt; im Einzelhandel ca. 28.000. Übersetzt ins Deutsche wurden 9.903 Bücher (vermutlich überwiegend aus Belletristik), und 2678 Lizenzen wurden ins Ausland vergeben. Der Absatz von E-Books auf dem Publikumsmarkt ist 2018 gegenüber dem Vorjahr um 12,7\%, der Umsatz um 9,3\% gestiegen.(Pressemitteilung des Börsenverein vom 15.02.2019 https://bit.ly/375xymW) 32,8 Millionen E-Books wurden 2018 verkauft. Damit machen E-Books 5,0 Prozent des Buchumsatzes am Publikumsmarkt aus. Das Ausleihen von E-Books erfolgt ebenfalls in erster Linie auf dem Publikumsmarkt und zwar über die öffentlichen Bibliotheken. Nach einer Studie im Auftrag des Börsenverein von Ende 2019 (https://bit.ly/2SnDEcV) leihen insgesamt 2,6 Millionen Menschen in Deutschland über die Onleihe (das ist das vom Börsenverein bereitgestellte Ausleihsystem) digital Bücher und andere Medien aus, insgesamt 1,9 Millionen leihen E-Books. Der Börsenverein interpretiert die Daten so, dass „die Onleihe die Kaufbereitschaft buchaffiner und kaufkraftstarker Zielgruppen am Buchmarkt“" schmälert. 
1.718 (davon Mathematik 348); Sozialwissenschaften: 12.734 (davon Soziologie 2.505, Recht 2.831); Technik, Medizin, angew. Wiss: 12.127 (davon Medizin 4.034, Management 2.668); Künste, Unterhaltung: 9.700; Literatur: 25.537.

Der durchschnittliche Preis für Bücher über alle Bereiche beträgt Euro 25,70. Die durchschnittlichen Preise für Bücher aus den einzelnen Fächern variieren stark, z. B. für Informatik 42,19, Bibliotheks-/Informationswissenschaft 50,06, Sprache/Linguistik 53,70, Naturwissenschaft 31,83, Unterhaltung 27,08, Literatur 16,5 (Angaben in €-Preisen).

Es ist heute schon nicht mehr riskant davon auszugehen, dass in absehbarer Zukunft, zumindest in Bildung und Wissenschaft, auch größere Texteinheiten wie Monographien, Lehrbücher, Proceedings etc. primär in elektronischer Form zugänglich gemacht werden. ${ }^{578}$ Auf dem Publikationsmarkt ist diese Tendenz heute schon deutlich zu erkennen (Daten dazu in FN 577). Bezüglich der Digitalisierung von Büchern in der Wissenschaft sind zweifellos Unterschiede in den Textsorten auszumachen. Am stabilsten sind wohl derzeit noch Festschriften, für die das traditionelle gedruckte Buch in der Tat der Normalfall ist. Proceedings, die Zusammenstellung der Beiträge von Fachkonferenzen, sind offensichtlich offener für die elektronische Veröffentlichung - sei es im Rahmen des hybriden Modells (Druck und e-Version) oder durch exklusive elektronische Veröffentlichung. Ein Beispiel für die exklusive Online-Version sind die Dagstuhl Seminar Proceedings. ${ }^{579}$ Seit 2014 werden die früher in der Reihe Dagstuhl Seminar Reports in gedruckter Form veröffentlichten Konferenzbeiträge aus dem Gebiet der Informatik nur noch als Dagstuhl Seminar Proceedings (DSP) online publiziert. Langzeitverfügbarkeit und Zitierfähigkeit sind dafür über den Online-Publikations-Server DROPS sichergestellt.

Für die sich abzeichnende Transformation in Richtung E-Books an Hochschulen sei als Beispiel auf die Bibliotheksbestände der FU-Berlin verwiesen. ${ }^{580}$ Die

578 Wenn Werke in der Zukunft weiter in gedruckter Form produziert werden, sei es exklusiv oder sekundär zur elektronischen Version, werden diese kaum noch nach dem bisherigen Modell finanziert werden können, das sich an der Anzahl der verkauften Exemplare orientiert. Autoren wissenschaftlicher Bücher, die auf dem klassischen Buchformat bestehen, könnte es passieren, dass sie für die entsprechenden Produktionskosten der Verlage vollständig aufkommen müssen. Einnahmen für diese Druckversionen sind über die APC hinaus für die Verlage kaum zu erzielen, wenn die Bibliotheken, bislang die Hauptabnehmen für gedruckte Bücher in der Wissenschaft, ihre Vermittlungsleistungen über die e-Version erbringen. Gedruckte Bücher könnten also ein Luxus für deren Liebhaber werden.

579 Dagstuhl Seminar Proceedings - https://bit.ly/2PSe7ZW.

580 FU-Berlin, ZEDAT - https://bit.ly/2qqd5tm. Die FU beschränkt den Zugang „,aus lizenzrechtlichen Gründen auf Rechner aus dem Campusnetz der lizenzgebenden Institution“. Allerdings besteht für FU-Angehörige die Möglichkeit, über einen ZEDAT-Account einen Zugang zu den 
Bibliothek hat insgesamt ca. 4 Mio gedruckte Bücher in ihren Beständen, dazu ca. 700.000 E-Books (durch Kauf oder Lizenz). Das macht mehr als ein Achtel des Gesamtbestandes aus. Trotzdem - obgleich so gut wie alle Texte/Wissensobjekte von wissenschaftlichen Autoren schon in digitaler Form erstellt werden, ist das Ergebnis deren Transformation in marktfähige Informationsprodukte derzeit primär noch das gedruckte produzierte Buch. Die online verfügbare elektronische Version ist derzeit noch das sekundäre Produkt. Dieses Verhältnis zwischen gedrucktem Buch und E-Version sollte sich - wie schon auf dem Publikumsmarkt zu erkennen (FN 577) sehr bald umdrehen. Speziell das elektronische Publizieren für Bücher bleibt noch ein „breites Experimentierfeld“581 für entsprechende Geschäfts-/Organisationsund Finanzierungsmodelle (zur Transformation auch der Buchmärkte in Richtung Open Access vgl. 14.11).

\subsection{Zum Geschenkmodell auf den Wissenschaftsmärkten}

Der Erfolg auf den kommerziellen Informationsmärkten beruht auch darauf, dass die Verlage für die wichtigste Leistung im Prozess des Öffentlichmachens von Wissen - das ist die Erstellung von Wissensobjekten durch die Wissenschaftler nichts bezahlen müssen. Wissenschaftler verschenken ihre Wissensobjekte i. d. R. den Verlagen. Allerdings erwarten sie als Gegenleistung, dass ihre Werke in attraktiver Form und in attraktiven Organen veröffentlicht werden. Das kann dann durchaus mit Blick auf ihre Karriere auch monetäre Folgen haben (vgl. Kap. 8). Die die Autoren finanzierenden öffentlichen Einrichtungen (Hochschulen, Länder, Bund, Förderorganisationen usw.) lassen das „Schenken“ weiter geschehen. Eine kostenpflichtige Lizenz für die kommerzielle Nutzung wurde dafür i. d. R. nicht verlangt - weder von den Autoren noch von den diese finanzierenden Einrichtungen. Die Honorare, wenn sie denn überhaupt (und nie an die Organisationen) gezahlt werden, sind kein Ersatz für eine zu erwerbende Lizenz. Dieses Geschäftsmodell des wissenschaftlichen Publizierens war im Grunde immer schon ein gewisses Ärgernis, aber ein in der Öffentlichkeit und auch wissenschaftsimmanent nicht beachtetes bzw. toleriertes Ärgernis. „Frieden“ war sozusagen auf den Wissenschaftsmärkten.

elektronischen Materialien der Bibliothek der FU-Berlin von extern (remote access) zu erhalten. Diese Lösung trägt dem Rechnung, dass eine Bibliothek zunehmend eine virtuelle Einrichtung ist und nicht bloß ein materielles Gebäude.

581 (Schmiede 2013) Wissenschaft+digitale Publikation: Das Publizieren bleibt „ein breites Experimentierfeld unterschiedlicher Kombinationen von gedruckter und digitaler Publikation, von kostenpflichtigem und Open-Access-Zugang, das je nach Fachkultur ganz unterschiedlich aussieht“. 
Frieden, solange die erbrachten Leistungen als spezielle und unverzichtbare Kompetenz der Verlage anerkannt wurden und solange die Preispolitik der Verlage die Nutzung des publizierten Wissens nicht behinderte - solange also die Bibliotheken, alleine oder im Verbund mit anderen, in der Lage waren, den Nutzungserwartungen aus der Wissenschaft und in der Ausbildung zu entsprechen. Davon kann heute nicht mehr die Rede sein. Dieses auf Geschenk beruhende Geschäftsmodell auf den wissenschaftlichen Zeitschriftenmärkten beschreiben (Ziegler 2019) und (Fyfe et al. 2017) wie folgt:

Verlage, so Ziegler „bekommen die Ergebnisse der mit Steuern finanzierten Forschung von der Wissenschaft geschenkt - in Form von druckfertigen Aufsätzen. Der Verlag leistet relativ wenig, nimmt den fertigen Aufsatz dann aber in Geiselhaft: der publizierte Aufsatz verschwindet hinter einer Bezahlschranke, das Geschenkte wird den interessierten und steuerzahlenden Bürgerinnen und Bürgern, der Wissenschaft und selbst den Autorinnen und Autoren also teuer zurückverkauft.“

„We are not the first“, so (Fyfe et al. 2017) „to wonder why academics continue to give their labour - as authors, referees and editors - to publishing firms that do not, in fact, circulate knowledge widely and affordably. The answer lies in a lack of detailed understanding among academics of the historical and economic forces at play in academic publishing; and in the success with which big publishers have learned how to make themselves apparently indispensable to the academic prestige economy.“

Die Kritik an dem Geschenkmodell ist aber nur ein Indiz dafür, dass in den letzten Jahren das Zusammenspiel der Akteure auf den Wissenschaftsmärken immer mehr als problematisch empfunden wurde. Verlage haben es zwar auch unter den veränderten Bedingungen noch für einige Zeit verstanden, sich, wie bislang, quasi als naturgegebener Partner von Bibliotheken und Endnutzer zu behaupten, so wie es viele Jahrhunderte lang der Fall gewesen war. Aber das konnte unter den veränderten informations- und kommunikationstechnologischen Rahmenbedingungen auf Dauer keinen Bestand haben. „Eigentlich”, so (Ziegler 2019) „können Wissenschaftlerinnen und Wissenschaftler die Arbeit der Verlage weitgehend selbstständig und unabhängig übernehmen.“ Mit „Arbeit“ ist hier das Publizieren gemeint. Ob sie das wirklich wollen, ist eine andere Frage.

Tatsächlich ist inzwischen umfänglich Endnutzersoftware z. B. zum Erstellen von Zeitschriften und damit für das Publizieren verfügbar, ${ }^{582}$ und es entstehen

582 Ein Beispiel: CeDiS (an der FU Berlin) (https://bit.ly/2ky804v) bietet mittels der Software OJS (Open Journal Systems) Hosting und Support für wissenschaftliche Fachzeitschriften (auch für Monographien). Ausführliche Informationen für „Herausgeber/innen von Zeitschriften“ auf der Open-Access-Plattform - https://bit.ly/2k8R8wf. Für Open-Access-Bücher bietet z. B. OAPEN Unterstützung zur Erstellung und Bereitstellung an. Die Publikationssoftware Open Monograph Press (OMP) wird zunehmend auch von Universitäten zur Unterstützung des E-Publishing für 
auch immer mehr offene, also nicht-proprietäre Zeitschriften. Herausgeber solcher Open-Access-Zeitschriften können einzelne Wissenschaftler sein, aber auch Fachverbände/-gesellschaften oder Institutionen der Autoren, in der Regel über die Bibliotheken der Hochschulen oder außeruniversitären wissenschaftlichen Einrichtungen. Auch beteiligen sich Verlage schon an solchen Open-Access-Vorhaben. Das hat aber bislang noch nicht zu dem geführt, was (Ziegler 2019) mit der Übernahme durch die Wissenschaft anmahnt. Dass dies von Seiten der Wissenschaft noch nicht flächendeckend getan wird, ist auch der Tatsache geschuldet, dass das Management von Zeitschriften und die Bereitstellung der Wissensobjekte auf den Informationsmärkten nicht zu den Kernaufgaben der Wissenschaft gehört. Es war über lange Zeit sehr bequem, auf die Leistungen der Verlage und den Dienstleistungen der Bibliotheken zu vertrauen. Heute scheint das kommerzielle Geschenk-/Verwertungsmodell nicht mehr akzeptabel zu sein - vor allem weil die Gegenleistungen nicht mehr als angemessen empfunden werden:

(1) Für die Öffentlichkeit wird immer weniger akzeptiert, dass das mit öffentlichen Mitteln produzierte Wissen i. d. R. kostenlos an die Verlage abgegeben wird und dass dann für die entstehenden Produkte hohe Nutzungsgebühren entrichtet werden müssen.

(2) Autoren sehen sich in ihrer informationellen Autonomie durch die Praxis kommerzieller Verwerter eingeschränkt und protestieren gegen die bisherige Praxis der vertraglich abgesicherten vollständigen und durch das Urheberrecht ermöglichten Übertragung der den Autoren zustehenden Verwertungsrechte in exklusive Nutzungsrechte der Verleger auf. Auch Förderorganisationen wie die DFG ermuntern Autoren, nicht länger ihre Verwertungsrechte komplett abzugeben (vgl. FN 564)

(3) Den Akteuren in Bildung und Wissenschaft in ihrer doppelten Eigenschaft als Produzenten und Nutzer von Wissen ist nicht mehr im ausreichenden Ausmaß der Zugriff auf die Informationsprodukte garantiert, die sie für das Schaffen neuen Wissens brauchen. Entsprechend baut sich zunehmend Widerstand (bis hin zu Boykottaufrufen) gegen Teile der Verlagswirtschaft auf (vgl. 14.6).

(4) Die Nutzungsbedingungen (aber auch die Gebühren) der kommerziellen Anbieter sind nicht mehr den Potenzialen elektronischer Medien angemessen. Entsprechend werden die hohen Gewinne, vor allem der großen internationalen Verlage, als unangemessen gesehen.

digitale Bücher eingesetzt, z. B. von der FU Berlin. Zu den auf Open Access ausgerichteten Universitätsverlagen vgl. FN 631 und FN 632. Ebenso haben viele Universitäten Publikationsfonds zur finanziellen Unterstützung von Open-Access-Publikationen eingerichtet (auch für die APC für Bücher). Vgl. die umfassenden Informationen zu Open-Access-Büchern auf der Plattform open-access.net - https://bit.ly/2Ozp4Ok. 
(5) Die Budgets der Mittlerinstitutionen wie Bibliotheken können nicht mehr mit den ansteigenden Kosten mithalten, die durch die aggressive und restriktive Preis- und Nutzungspolitik im Rahmen der Subskriptionsmodelle entstanden sind, begünstigt durch die Quasi-Monopolstruktur großer Verlage.

(6) Bibliotheken steigen daher immer mehr aus den Verträgen vor allem mit den großen Verlagen wie Elsevier aus und suchen, unterstützt von den Wissenschaftsorganisationen, nach neuen Formen der Finanzierung der Verlagsangebote (z. B. im Rahmen von DEAL; vgl. ausführlich dazu in 14.8).

(7) Das Publikationsmonopol der Verlage wird insgesamt nicht mehr anerkannt. Der Einsatz von Technologien für das Öffentlichmachen/Publizieren und Verbreiten von Informationsobjekten ist nicht länger das exklusive Kompetenzprofil für kommerzielle Verlage. Öffentlichmachen/Publizieren von erarbeitetem Wissen kann durch die umfassende Verfügbarkeit von entsprechenden Technologien aus der Wissenschaft, zusammen mit den Bibliotheken und Fachgesellschaften, selbst organisiert werden.

(8) Wissenschaft entwickelt unabhängig von den rechtlichen Vorgaben neue Formen der Produktion, des Öffentlichmachens, der Verteilung und Nutzung von Wissen und Information.

Das hier skizzierte „Geschenkmodell“ könnte aber auch ganz anders bestimmt werden. Die Geschäftsmodelle der großen Player des „digitalen Kapitalismus“ (Staab 2019) beruhen überwiegend auf einem etwas anderem Geschenkmodell, für das die Bezeichnung „Freemium“ verwendet wird. Hier gilt i. d. R. das, was auch in dieser Arbeit als Nutzungsfreiheit bezeichnet - zumindest gilt das für die meisten Produkte und Dienstleistungen von Google und Facebook. Das ist nicht die Freiheit, die hier gemeint ist; denn die freie Nutzung wird in diesem Geschenkmodell dennoch bezahlt, allerdings mit einer anderen Währung, nämlich mit den Daten der Nutzung und der Nutzer, die für den viele Milliarden umfassenden Gewinn in realer Währung verwendet werden. ${ }^{583}$ Freemium bedeutet also, dass für das primäre Basismodell auf Einnahmen verzichtet wird - die Nutzung also quasi geschenkt wird. Gewinne werden mit Anderem erzielt, wie bei Google und Facebook durch auf den Nutzungsdaten beruhender Werbung. Wem der Privatheitsschutz der persönlichen Daten das

583 Alphabet Inc., das Mutterunternehmen von Google, setzte im zweiten Quartal von 2019 rund \$38,94 Mrd um, mit einem Gewinn von \$ 9,94 Mrd. Das war eine Steigerung des Quartalsumsatzes vom 19\% - Quelle: statista: Umsatz von Google weltweit vom 4. Quartal 2014 bis zum 4. Quartal 2019 - https://bit.ly/2PGEl1u. Der Umsatz von Facebook betrug im dritten Quartal von 2019 ca. \$17,7 Mrd und erbrachte einen Gewinn von \$ 6.1 Mrd (durch monatlich aktive 2,45 Mrd Facebook-Nutzer) Daten aus ZEIT 31.10.2019 - https://bit.ly/38gVxkb. 
höhere Gut ist, sollte das Geschenk als „vergiftet“ bezeichnet werden; aber kaum jemand verzichtet konsequent auf die Nutzung dieser Dienste. Monetäre Gewinne im Freemium-Modell können aber auch dadurch erzielt werden, dass für die die Basisprodukte ergänzenden informationellen Mehrwerte oder dass für die freien Basisprodukte in anderer medialer Gestalt bezahlt werden muss, z. B. elektronisch frei und gedruckt kostenpflichtig (oder umgekehrt). Kann das Freemium-Modell, das letztlich immer auch den kommerziellen Gewinnerwartungen entspricht, auf Produkte und Dienstleistungen auf den Wissenschaftsmärkten übertragen werden? Bislang besteht das traditionelle kommerzielle Verwertungsmodelle weiter, das auf Verkauf und/oder Lizenz für möglichst viele Exemplare bzw. möglichst viele Lizenzabnehmer und eng definierte Lizenznutzungsbedingungen beruht und auf das das Urheberrecht unterstützend reagiert. Im Folgenden wird beispielartig darauf eingegangen, wie sich die Wissenschaft und auch die Wissenschaftsorganisationen gegen dieses Modell wehrt.

\subsection{Wissenschaft wehrt sich}

In 3.5 wurde schon darauf eingegangen, dass sich seit der Jahrtausendwende der Widerstand gegen das kommerzielle Verwertungsmodell geregt hat. Fassen wir das kurz zusammen:

Der Wissenschaftsrat sprach schon $2001^{584}$ von den „Veränderungen der Rollenverteilung in der Publikations- und Informationskette vom Autor bis zum Nutzer wissenschaftlicher Informationen” und von entsprechenden ,Veränderungen der Rollenverteilung in der Publikations- und Informationskette vom Autor bis zum Nutzer wissenschaftlicher Informationen.“ Das damalige Forschungsministerium des Bundes sprach sich für den uneingeschränkten Zugriff auf das publizierte Wissen aus. Auf dem Weltgipfel der UN zur Informationsgesellschaft (2003 in Genf und 2005 in Tunis) wurde, auch mit Blick auf die Entwicklungsländer, der freie Zugriff zu einem globalen Thema. Es gab immer mehr Initiativen und Eingaben auf EU-Ebene, z. B. die 2007 von vielen Tausend Personen und Institutionen unterzeichnete Petition an die Europäische Kommission:

\footnotetext{
Open access to the published scientific literature is one of the most desirable goals of our current scientific enterprise. Since most science is supported by taxpayers it is unreasonable that they should not have immediate and free access to the results of that research.
}

584 Wissenschaftsrat in seinen „Empfehlungen zur digitalen Informationsversorgung durch Hochschulbibliotheken“ 13.7.2001 (Drs. 4935/01) - https://bit.ly/2P5TbzJ. 
2009 wurde von ca. 25.000 Personen eine von Lars Fischer an den Deutschen Bundestag gerichtete Petition unterzeichnet. Diese hatte das Ziel zu erreichen, dass wissenschaftliche Publikationen, die aus öffentlich geförderter Forschung hervorgehen, allen Bürgern kostenfrei zugänglich sein müssen (vgl. FN 68). Bei einer 2011 vom Aktionsbündnis durchgeführten Umfrage wurde die Frage gestellt: „Sollte Wissen, das unter Einsatz öffentlicher Mittel gewonnen wurde, Ihrer Meinung nach für jedermann für seinen persönlichen Bedarf frei verfügbar sein?“ Von 91,7 \% der Antworten wurde dies bejaht (vgl. FN 70). Ergänzend dazu zwei Beispiele für den aktiven Widerstand:

a) The cost of knowledge. Der 2012 vom Mathematiker William Timothy Gowers initiierten Aufruf "The cost of knowledge" gegen den Wissenschaftsverlag Elsevier ${ }^{585}$ ist nur ein Beispiel für die zahlreichen öffentliche Proteste aus der Wissenschaft gegen die restriktive Preis-, Distributions- und Nutzungspolitik durch die Oligopole der großen Zeitschriftenverlage. Den Protest-Aufruf haben 17.641 Forscher unterzeichnet (keineswegs nur Mathematiker), und viele von ihnen haben ausdrücklich erklärt, nicht weiter für Elsevier zu arbeiten: “won’t publish; won’t referee; won’t do editorial work". In den Erläuterungen zu "Cost of knowledge” wurde auf den technologischen Wandel verwiesen, der den Aufwand für das Publizieren drastisch verringert hat und der damit das Monpol der Verlage für das Publizieren in Frage stellt:

the cost of journal publishing has gone down because the cost of typesetting has been shifted from publishers to authors and the cost of publishing and distribution is significantly lower than it used to be. By contrast, the amount of money being spent by university libraries on journals seems to be growing with no end in sight. Why do mathematicians contribute all this volunteer labor, and their employers pay all this money, for a service whose value no longer justifies its cost?

Zwar wurde Elsevier als der besonders aggressiv mit hoher Preispolitik operierende Verlag angegriffen ("Elsevier is an exemplar of everything that is wrong with the current system of commercial publication of mathematics journals"), aber in Frage gestellt wurde die gesamte herkömmliche kommerzielle Publikationswelt. Aber es wurden auch Antworten darauf gesucht, die den Möglichkeiten des Internets besser entsprächen. Es gibt nicht die eine Lösung. Auch das Zusammenspiel von Open-

585 Vgl. Forscheraufstand gegen Wissenschaftsverlag. Mathematiker: Verlage lassen Unibibliotheken ausbluten. Deutschlandfunk 19.3.2012 - https://bit.ly/2kArJvr; vgl. (Scholz 2018) Wem gehört das Wissen? Vgl. die hier erwähnten Petitionen an die Politik zugunsten eines freien Umgangs mit öffentlich finanziertem Wissen (FN 68 und 69). Vgl. (Czepel 2015) Fünf Konzerne regieren die Wissenschaft - mit Bezug auf (Larivière et al. 2015) The Oligopoly of Academic Publishers. 
Access- und traditionellen Zeitschriften wurde, im Sinne der Kompatibilitäts-These der beiden Märkte, nicht ausgeschlossen:

Some people would like to see the journal system eliminated completely and replaced by something else more adapted to the internet and the possibilities of electronic distribution. Others see journals as continuing to play a role, but with commercial publishing being replaced by open access models. Still others imagine a more modest change, in which commercial publishers are replaced by non-profit entities such as professional societies (...) or university presses; in this way the value generated by the work of authors, referees, and editors would be returned to the academic and scientific community. These goals need not be mutually exclusive: the world of mathematics journals, like the world of mathematics itself, is large, and open access journals can coexist with traditional journals, as well as with other, more novel means of dissemination and evaluation.

b) Sci-Hub. Der Protest gegenüber restriktiver Preispolitik und damit eingeschränkte Nutzungsmöglichkeiten kann aber auch viel radikalere Züge annehmen. Die Online- Plattform Sci-Hub wurde im September 2011 von der kasachischen Neurowissenschaftlerin Alexandra Elbakyan gegründet. ${ }^{586}$ Programmatische Basis dieser Plattform ist die Überzeugung, dass jedem Nutzer der barrierefreie, kostenlose Zugang zu allen veröffentlichten wissenschaftlichen Inhalten zustehe. ${ }^{587}$ Elbakyan hatte Softwarelücken sowohl in den Bibliotheken der Universitäten und Forschungseinrichtungen sowie von Informationsdiensten wie ScienceDIrect von Elsevier ausgenutzt und so mehr als 60 Millionen wissenschaftliche Artikel heruntergeladen und zur freien Nutzung über das Internet bereitgestellt. ${ }^{588}$ Sci-Hub bzw. Elbakyan sind mehrfach verklagt und auch verurteilt worden. Trotzdem bleibt der Zugriff darauf über wechselnde Domain-Namen erhalten, wenn auch jetzt mit einigem Suchaufwand verbunden. Die Verbote bzw. der offensichtliche Verstoß gegen Urheberrechtsgesetze haben die intensive Nutzung durch Wissenschaftler nicht eingeschränkt. 2017 konnte über Sci-Hub auf 62 Mio Artikel zugegriffen werden; das macht etwa $85 \%$ der kostenpflichtigen Artikel aus „paywalled scholarly journals" aus, wenn auch nur 69\% aller insgesamt publizierten Artikel. Schon 2016 wurden 200.000 Anfragen an Sci-Hub gestellt - pro Tag. Nach einer aktuellen Studie von (Till et al. 2019) ${ }^{589}$ verzeichnete Sci-Hub 27.8 Mio erfolgreich bearbeitete Download-Anfragen zwischen September 2015 und Februar 2016.

586 Vgl. den Artikel Sci-Hub in der Wikipedia - https://bit.ly/2lIAuUm.

587 Die allgemeine, obgleich nicht rechtlich bindenden Referenz dafür ist Artikel 27 der Allgemeinen Erklärung der Menschenrechte der Vereinten Nationen von Dezember 1948: „Jeder hat das Recht, am kulturellen Leben der Gemeinschaft frei teilzunehmen, sich an den Künsten zu erfreuen und am wissenschaftlichen Fortschritt und [dessen Errungenschaften teilzuhaben.“

588 Die folgenden Daten sind dem Wikipedia-Artikel zu Sci-Hub entnommen (vgl. FN 586).

589 (Till et al. 2019) Who is pirating medical literature? 
Aus der Sicht der kommerziellen Verlagswelt ist Sci-Hub illegal, eine Piraterie, welche ihre traditionelle Geschäftsgrundlage, aber auch Aktivitäten für kommerzielles Open Access untergrabe. Sci-Hub ist auch mit bestehenden Urheberrechtsregelungen zweifellos nicht vereinbar. Aber was sagt das aus über die Akzeptanz und Beachtung von rechtlichen Vorgaben? Geschäftsmodelle und juristische Regelungen, die von den auf sie bezogenen Akteuren im großen Stil nicht bedient und nicht beachtet werden, laufen leer. Das geht einher mit der eingangs aufgestellten These, dass beides, Markt und Recht, nicht auf Akzeptanz rechnen können, wenn sie mit dem entwickelten moralischen Bewusstsein für den Umgang mit Wissen und Information nicht übereinstimmen. Damit ist nicht die Legitimität der Nutzung von Sci-Hub institutionalisiert, und jeder Nutzer ist für die von ihm in Anspruch genommene Nutzung verantwortlich, auch wenn er sie als Zivilcourage verstehen mag. Sci-Hub steht in die Tradition dessen, was früher die Napsterisierung von Wissen als Protest gegen dessen fortschreitende Venterisierung (Kommerzialisierung von Wissen) genannt wurde. Vielleicht ist es so, dass sich nichts ändert, wenn nicht durch außerhalb des Systems stehende Handlungen die problematisch gewordenen Regelungen dieses Systems „kreativ zerstört“ werden. Eine Zerstörung wie durch Sci-Hub ist sicher nicht nachhaltig nachhaltig aber vielleicht die Auswirkung auch auf das Recht.

Die die kommerzielle Verwertung in Frage stellende Bereitstellung von Informationsobjekten durch Sci-Hub steht den Bibliotheken und den offiziellen Wissenschaftsorganisationen nicht zur Verfügung. Sie können ihren Protest aber auch anders artikulieren. Mehr als 200 Bibliotheken in Hochschulen und außeruniversitären Wissenschaftseinrichtungen hatten Ende 2017 ihre Verträge mit Elsevier gekündigt, um sich vor allem von „knebelnden“ Subskriptionskosten für gebündelte Zeitschriften-Abonnements frei zu machen. Verschiedentlich wurde von den Bibliotheken versucht, für ihre Mitglieder einen kostenlosen Service einzurichten, durch den einzelne Elsevier-Artikel über die Bibliothek bestellt werden können. ${ }^{590}$ Diese können dazu den Direktbestellungsdienst über ScienceDirect verwenden. Elsevier stellte, allerdings befristet, trotz der Befristung für eine Weile den vollen Service bereit. Wissenschaftsorganisationen müssen auf Verhandlungen setzen, um die Bibliotheken weiter in die Lage zu versetzen, die Informationsbedürfnisse ihrer Klientel zu befriedigen. In Deutschland hat vor allem die DFG schon seit einiger Zeit auf Nationallizenzen gesetzt, um die Bibliotheken von der restringie-

590 Vgl. Uni-Bibliothek Bremen - https://bit.ly/2lKrwWH. Der Prozess bis zur Auslieferung (nur über Papier erlaubt, nicht elektronisch) soll in der Regel 7 Tage dauern Ein weiteres Beispiel für neue Formen der Direktlieferung (wenn kein Lizenzvertrag besteht): Bibliothek der Universität Konstanz: https://bit.ly/2NxgHCB. 
renden Preispolitik der Verlage zu entlasten. ${ }^{591}$ Die weiteste Perspektive eröffnet sich allerdings durch die Verhandlungen im Rahmen von DEAL (vgl. 14).

\subsection{Zu den Open-Access-Zeitschriftenmärkten}

Die Leitidee, dass der Zugang zum publizierten Wissen für jedermann frei zugänglich nutzbar sein soll, wird zwar immer noch langsam, aber doch beständig zum Default des wissenschaftlichen Publizierens - bislang in erster Linie für die Arbeiten in wissenschaftlichen Zeitschriften. Dieser Trend ist Realität und wird durch die Dokumentation von DOAJ (der zentrale Nachweisdienst für Open-AccessZeitschriften) bestätigt. DOAJ wies für Oktober 20117.183 Open-Access-Zeitschriften nach, für Anfang August 2019 13.597. Von diesen Zeitschriften sind 10.650 auf Artikelebene suchbar. Insgesamt sind 4.172.826 Open-Access-Artikel in insgesamt 131 Ländern vorhanden. Verglichen mit den in 14.4.1 angegebenen Daten zu den online verfügbaren Zeitschriftenartikeln insgesamt machen die Open-Access-Artikel allerdings nur ca. $4 \%$ aus.

Der oft noch niedrige Impact Factor (IF) von Open-Access-Zeitschriften ist dadurch bedingt, dass sie i. d. R. noch neueren Datums sind. Artikel in OpenAccess-Zeitschriften haben aber hohe Wahrnehmungs- und damit Zitierungswahrscheinlichkeit, ihre Autoren also einen „Zitiervorteil“. Der Nationale Open-AccessKontaktpunkt (ein von der Allianz der deutschen Wissenschaftsorganisationen gefördertes und an der Universität Bielefeld angesiedeltes Projekt) belegt dies auf Grund mehrerer Studien:

Generell lässt sich sagen, dass Open-Access-Zeitschriften eine höhere Sichtbarkeit haben und mittlerweile so etabliert sind, dass sie unter den Top-Zeitschriften vieler wissenschaftlicher Fachrichtungen zu finden sind..$^{592}$

591 Auf der Website der DFG für Nationallizenzen heißt es: "Um die Versorgung mit elektronischer Fachinformation an deutschen Hochschulen, Forschungseinrichtungen und wissenschaftlichen Bibliotheken nachhaltig zu verbessern, finanziert die Deutsche Forschungsgemeinschaft (DFG) seit 2004 den Erwerb von nationalen Lizenzen für elektronische Medien im Rahmen ihres Förderprogramms „Überregionale Literaturversorgung und Nationallizenzen“. (https://www.nationallizenzen.de/) Ziel ist es, Wissenschaftlern, Studierenden und wissenschaftlich interessierten Privatpersonen den Zugang zu Datenbanken, digitalen Textsammlungen, elektronischen Zeitschriften und E-Books zu ermöglichen. Diese Lizenzen können von Einzelpersonen und Institutionen erworben werden. Eine Liste der Nutzungsangebote unter DFG Nationallizenzen https://bit.ly/2sAQg7S.

592 oa2020.de: Meist-zitierte Open-Access-Zeitschriften - https://bit.ly/2kosAQ5. 
Der Anstieg an Open-Access-Zeitschriften hat sich inzwischen verlangsamt, aber über die gesamte hier angegebene Periode ist das immer noch ein durchschnittlicher Zuwachs von ca. 2 Zeitschriften pro Tag weltweit. Für Artikel in Open-Access-Zeitschriften gelten die beiden zentralen „Qualitäts“sicherungsverfahren, vor allem das klassische Peer Reviewing durch die Wissenschaft und bislang auch der IF als Exzellenznachweis für Zeitschriften. Da die meisten Open-Access-Zeitschriften noch nicht lange existieren (selten mehr als 10 Jahre), haben sie es schwerer als die traditionellen kommerziellen Zeitschriften, einen hohen IF zu erreichen. Das scheint aber nur eine Frage der Zeit zu sein.

Auf jeden Fall müssen für Open-Access-Zeitschriften die gleichen hohen Qualitätsansprüche gelten wie sie in der traditionellen Publikationsumgebung sich eingebürgert haben. Das wird schon in den verschiedenen Open-Access-Deklarationen festgehalten. Allerdings ist in letzter Zeit die Qualitätssicherung dadurch problematisch geworden, dass sich auf den Informationsmärkten Zeitschriften unter dem Etikett „Open Access“ etabliert haben, die nicht die Standards wissenschaftlicher Qualitätssicherung oder redaktioneller Bearbeitung erfüllen und die gegen entsprechende Gebühren quasi unbesehen alles veröffentlichen, was ihnen angeboten wird. Allein das Angebot „Open Access“ lockt offensichtlich in beträchtlichem Umfang viele Wissenschaftler, in diesen pseudowissenschaftlichen Raubverlagen zu veröffentlichen. Dafür hat sich auch der Begriff Fake-Science oder Junk-Science eingebürgert, mit beträchtlichem Schaden für das Ansehen von Wissenschaft. ${ }^{593}$ DOAJ hat versucht, solche Open-Access-Zeitschriften herauszufiltern. So ist der Rückgang der 10.222 bei DOAJ nachgewiesenen Open-Access-Zeitschriften zum 7.2.2015 auf 9.325 zum 24.11.2016 vermutlich dadurch zu erklären, dass entsprechende Fake-Open-Access-Zeitschriften herausgenommen wurden. Zur Verminderung der Unsicherheit über die Qualität von Open-Access-Zeitschriften kann über den Nationalen Open-Access-Kontaktpunkt (OA2020-DE) eine Liste von 800 in DOAJ nachgewiesenen Open-Access-Zeitschriften durchsucht werden, die einen überdurchschnittlichen SNIP-Faktor aufweisen. Der SNIP-Faktor (source normalized impact per paper) ist eine zum IF alternative Journalmetrik, die den durchschnittlichen Zitationseinfluss der Veröffentlichungen einer Zeitschrift misst. ${ }^{594}$

Die Open-Access-Entwicklung wird schrittweise auch von der Verlagswelt akzeptiert - entweder mit eigenen, also selbst entwickelten Open-Access-Zeitschriften oder durch Übernahme schon bestehender, aus der Wissenschaft entwickelten Open-Access-Zeitschriften (flipped journals) oder die Herausgabe eigener Open-

593 (Walger/Walger 2019) Vom Schein des Rechten getäuscht.

594 Vgl. University of Maryland Research Guides: Bibliometrics and Altmetrics: Measuring the Impact of Knowledge - https://bit.ly/2SD2NjU. 
Access-Bücher. Als Zwischenform kommen auch hybride Modelle sowohl für die Zeitschriften als auch für größere Objekte wie Bücher zum Einsatz. Hierbei können die Autoren von Artikeln oder Büchern entscheiden, ob sie ihre Verwertungsrechte zur kommerziellen Nutzung an Verleger abtreten oder ob sie, in der Regel durch Zahlung einer Gebühr (APC), das Open-Access-Publizieren ihrer Arbeit ermöglichen. So entstehen hybride Zeitschriften, bei denen ein Teil frei herunterladbar ist, der andere aber nur entweder durch Direktkauf oder über das erworbene Abonnement ihrer Bibliotheken. Dieses Abonnement müsste dann um den Anteil der Open-Access-Artikel in der Zeitschrift reduziert werden. Das ist kein einfaches und kein leicht transparent zu machendes Abrechnungsverfahren. Ob das ein zukunftsträchtiges Modell ist, kann zumindest für Zeitschriften bezweifelt werden. Durch DEAL soll das korrigiert werden.

Alle großen Zeitschriftenverlage sind in der Tat inzwischen auf breiter Front in das kommerzielle Open-Access-Publizieren eingestiegen (Daten von Mitte 2019):595

Wiley ${ }^{596} 110$ Open Access-Zeitschriften, dazu 1420 Zeitschriften nach dem hybriden Ansatz; SpringerNature ${ }^{597} 600$ Open Access-Zeitschriften, dazu 1700 nach dem hybriden Ansatz; Elsevier ${ }^{598}$ ca. 170 Open Access- Zeitschriften, dazu 1850 nach dem hybriden Ansatz; Taylor \& Francis ${ }^{599} 250$ Open Access-Zeitschriften, dazu 2300 nach dem hybriden Ansatz.

Das gesamte kommerzielle Open-Access-Geschäftsmodell bei den Zeitschriften beruht (derzeit) darauf, dass Verlage sich ihre Leistungen (und ihre Gewinne) von den Autoren direkt bzw. mehr und mehr von ihren Institutionen, von Fachverbänden, von Förderinstitutionen oder den staatlichen Trägern der Hochschulen und externen Forschungseinrichtungen bezahlen lassen. Dadurch werden die verlagseigenen Open-Access-Zeitschriften finanziert - bislang in erster Linie über die APC-Gebühr. APC werden i. d. R. nicht von den Autoren selbst entrichtet, sondern durch die zugeordneten Institutionen. Auch Förderorganisationen übernehmen

595 Der Grund für den Einstieg der Verlage in das Open-Access-Publizieren liegt vermutlich nicht nur darin, dass wegen der öffentlichen Finanzierung der Open-Access-Arbeit der Verlage eine gesichertes Geschäftsmodell entstanden ist (s. unten), sondern auch darin, dass sie sich durch die weiterhin bestehende Zuständigkeit für Open-Access-Objekte die Basis für die Entwicklung von informationellen Mehrwerten und neuen auf Wissenschaft und Bildung gerichteten Dienstleistungen sichern wollen. (vgl. 14.8)

596 Wiley OA- https://bit.ly/2m770A5.

597 Springer Nature OA - https://bit.ly/2lCjFe3.

598 Elsevier OA - https://bit.ly/2m5g6x5. Elsevier geht davon aus, dass $20 \%$ aller Artikel, die jedes Jahr verfasst werden, über Open Access veröffentlicht werden- Elsevier hatte 2016420.000 Artikel veröffentlicht, davon mehr als 20.000 im Open-Access-Format. Der Elsevier-Service ScienceDirect erlaubt direktes Suchen nach OA, jetzt auch in SCOPUS.

599 Taylor \& Francis OA - https://bit.ly/2z2RNCI. 
ganz oder teilweise die APC, falls eine Publikation ein Ergebnis der Förderung ist. ${ }^{600} \mathrm{APC}$ wird für alle Wissenschaftler in einer Wissenschaftsorganisation von dieser ganz oder teilweise übernommen. ${ }^{601} \mathrm{APC}$-Gebühren streuen breit ${ }^{602}$ von 0-APC, über wenigen hundert $\$$ bis über $\$ 5.000$ für sehr teure Zeitschrift wie Cell. APC kann pauschal über einen Vertrag eines Verlags vereinbart werden, z. B. durch eine Hochschule. Deren Bibliothek finanziert, d. h. alle Angehörigen dieser Einrichtungen können dann in den Zeitschiften dieses Verlags Open Access publizieren. ${ }^{603}$ Auch kann ein Verbund von fachspezifischen Institutionen mit Verlagen die Open-Access-Produktion/-Nutzung für alle Wissenschaftler auf dem Fachgebiet aushandeln (und damit auch die weltweite Open-Access -Nutzung), so z. B. das SCOAP-Modell für die Hoch-Energie-Physik. ${ }^{604}$

Im Rahmen der DEAL-Verhandlungen wurde ein Finanzierungsmodell über eine PAR-Gebühr vereinbart. PAR ist mehr als nur ein institutionalisiertes APC, denn über PAR im Rahmen von DEAL wird nicht nur das Open-Access-Publizieren finanziert, sondern auch der (allerdings nicht umfassende) lesende Zugriff auf die kommerziellen Zeitschriftenbestände. Aber auch für diese Gebühr trifft zu, dass den Verlagen die Kosten für die Umwandlung der Wissensobjekte in Informationsobjekte (einschließlich des erwarteten Gewinns) von der Öffentlichkeit erstattet werden allerdings mit dem Effekt, dass dadurch die Nutzung weitgehend frei wird. Das ist das neue Modell, das den Verlagen erlaubt, auch auf den Publikationsmärkten weiter aktiv zu bleiben. Dazu ausführlicher im folgenden Abschnitt.

600 Beispiel: Die DFG hat bereits 2010 „,eine einheitliche Preisdeckelung für Open-Access-Artikelgebühren im Programm Open Access Publizieren eingeführt. Es bestehen DFG-Förderprogramme für die institutionelle Abdeckung von Publikationsgebühren sowie für Lizenzverträge, die OpenAccess-Komponenten enthalten.“ - https://bit.ly/2Humea5.

601 Beispiel: Die Max Planck Digital Library (MPDL) unterstützt das Open-Access-Publizieren der MPI-Wissenschaftler durch Hinweise, wie die finanziellen APC-Anforderungen ganz oder teilweise übernommen werden, z. B. „All Max Planck authors are granted a $50 \%$ discount on the article processing charges for all genuine Open Access journals“ - https://bit.ly/2km7gKZ.

$602 \mathrm{Vgl}$. Angaben der University of Cambridge, UK - https://bit.ly/2Avdnl0.

603 Beispiel: Die Universität Göttingen (inklusive der Universitätsmedizin Göttingen) wurde Mitglied in SpringerOpen. Autoren zahlen keine APC für das Publizieren in den Zeitschriften von BioMed Central, Chemistry Central oder SpringerOpen. Die Rechnungsabwicklung übernimmt zentral die SUB Göttingen. Zeitgleich mit der Veröffentlichung in einem entsprechenden SpringerOpen Journal wird der Artikel über GoeScholar, d.i. der Publikationsserver der Universität Göttingen, zugänglich gemacht - https://bit.ly/2k5nisj.

$604 \mathrm{SCOAP}^{3}$ is a one-of-its-kind partnership of over three thousand libraries, key funding agencies and research centers in 44 countries and 3 intergovernmental organisations. Working with leading publishers, $\mathrm{SCOAP}^{3}$ has converted key journals in the field of High-Energy Physics to Open Access at no cost for authors. SCOAP ${ }^{3}$ - Sponsoring Consortium for Open Access Publishing in Particle Physics -https://scoap3.org/. 


\subsection{Die Transformation in Richtung Open-Access-Publizieren am Beispiel Deal}

International treibt OA2020, in Übereinstimmung mit den Zielen von Coalition $\mathrm{S}$ (vgl. 11.3.6), die Open-Access-Entwicklung voran. ${ }^{605}$ Das strategische Ziel von OA2010 ist es, die Transformation der Märkte in Richtung Open-Access-Publizieren dadurch zu erreichen, dass mit den Verlegern Übereinkommen (transformative agreements) getroffen werden, durch welches das Subskriptionsmodell für Zeitschriften(bündel) durch ein neues, Open-Access-spezifisches Geschäftsmodell ersetzt werden soll. Durch die Öffentlichkeit finanziert werden nur die Kosten für die Erstellung und die öffentliche Zugänglichmachung von Informationsobjekten durch die Verlage. Die Nutzung selbst ist frei. Das ist ein wesentlicher Beitrag, um die hier verfolgte Leitidee der Nutzungsfreiheit zu erreichen - allerdings kann das dafür zum Einsatz kommende Finanzierungsmodell durchaus als problematisch angesehen werden.

In Deutschland verfolgen auch die in der Allianz zusammengefassten Wissenschaftsorganisationen seit 2016 (vgl. FN 605) über das Vorhaben DEAL ein ähnliches Ziel, nämlich das bisherige Standard-Subskriptionsmodell für wissenschaftliche Literatur durch ein publikationsbasiertes Open-Access-Modell abzulösen. An DEAL sind derzeit 700 wissenschaftliche Einrichtungen in Deutschland beteiligt. Als Einstieg soll durch DEAL ${ }^{606}$ über bundesweite Lizenzverträge mit den großen Wissenschaftsverlagen ein „deal“ abgeschlossen werden, um sukzessive dieses allgemeine Ziel zu erreichen, ${ }^{607}$ konkret (hier paraphrasiert):

(1) Alle an DEAL beteiligten Einrichtungen soll dauerhafter Volltextzugriff auf das gesamte Zeitschriften-Portfolio der ausgewählten Verlage garantiert werden.

(2) (Peer Review bewertete) Publikationen von Autoren aus deutschen Einrichtungen sollen automatisch nach Open Access-Prinzipien frei geschaltet werden (i. d. R. ergänzt um die CC-BY-Lizenz).

(3) Die Vergütung an die Verlage soll durch ein einfaches, zukunftsorientiertes Berechnungsmodell erfolgen, das sich am Publikationsaufkommen orientiert.

605 Grundlegend für die Transformation des Subskriptionsmodells in ein Open-Access-Geschäftsmodell ist die Arbeit von (Schimmer/Geschuhn/Vogler 2015) Disrupting the subscription journals. 606 Projekt DEAL - https://www.projekt-deal.de/.

607 Der Börsenverein des deutschen Buchhandels hatte vor dem Bundeskartellamt gegen das Vorhaben DEAL geklagt, da dadurch in die Märkte für E-Zeitschriften missbräuchlich eingegriffen würde: Es gebe keinen transparenten Auswahlprozess, so dass Fachbuchhändler und kleinere Verlage außen vor seien. „Das Kartellamt“, so der Börsenverein, „lehnte eine Verfahrenseinleitung ab. Das Argument: Die Nachfragebündelung liege im Bagatellbereich, da unterhalb eines Marktanteils von 15 Prozent liegend (weltweiter Nachfragemarkt entscheidend). “ Börsenblatt 13.6.2017 https://bit.ly/2uq81GI. 
(4) Die einzelnen an Deal beteiligten Einrichtungen sollen finanziell entlastet werden, vor allem durch Befreiung vom Subskriptionsmodell.

Während es mit den Verlagen Wiley Anfang $2019^{608}$ und Springer Nature im August $2019^{609} \mathrm{zu}$ (befristeten) Lösungen gekommen ist, konnte mit Elsevier bislang (Stand 9/2019) keine Einigung erreicht werden, da Elsevier nicht bereit war, die zentralen DEAL-Ziele zu übernehmen, vor allem eine erweiterte Open-Access-Nutzung, das freie Lesen kommerzieller Bestände und - mit Blick auf zukünftige Verwertungsformen - die Aufgabe der exklusiven Nutzungsrechte. In den bislang getroffenen Vereinbarungen sind z.B. die folgenden Regelungen mit Springer Nature vorgesehen ${ }^{610}$ - vergleichbar denen mit Wiley. ${ }^{611}$

(1) Autoren, die an den DEAL-Einrichtungen arbeiten (Status "submitting corresponding author" ${ }^{12}$ ) können ihre Artikel nach Open Access-Prinzipien in den aktuell rund 1.900 Springer- bzw. den 1.420 Wiley-veröffentlichen Subskriptionszeitschriften veröffentlichen. Unklar, ob das von der Menge her begrenzt ist - die Rede ist von jährlich rund 13.000 Artikeln bei Springer bzw. 10.000 bei Wiley. Im Wiley-Vertrag heißt es dazu: „Die jährlichen Kosten des Wiley-DEAL-Vertrages bemessen sich an der Anzahl der jährlich publi-

$\mathbf{6 0 8}$ Wiley-Vertrag 15.1.2019 - https://bit.ly/3btmWSk. Ab dem 22.2.2019 konnten Autoren aus den Vertragspartnerinstitutionen gegen Zahlung der PAR Artikel in Wiley's Gold Open Access journals veröffentlichen, ab dem 1.7.2019 auch Artikel in Wiley's Hybrid-Zeitschriften. Erwartet werden ca. 10.000 jährlich in Wiley-Zeitschriften zu publizierende Artikel, die entsprechend Open Access unter einer freien Lizenz genutzt werden können. Für jeden dieser Artikel muss die PAR von $€ 2.750$ gezahlt werden.

609 Vgl. DEAL Springer Nature 22.8.2019 - https://bit.ly/2vYXnbk. Das Memorandum of Understanding (MOU) wurde am 22. August 2019 unterzeichnet - https://bit.ly/2LfnChV.

610 Die vollständigen Texte der Memoranden bzw. die gültigen Verträge waren zur Zeit der Fertigstellung dieser Arbeit noch nicht öffentlich einsehbar. Es kann daher sein, dass einige Aussagen hier korrigiert werden müssen oder dass sich inzwischen die Vereinbarungen geändert oder konkretisiert haben. Entsprechend den Eckpunkten von DEAL berechtigt die Vereinbarung „alle wissenschaftlichen Autorinnen und Autoren aus rund 700 deutschen Wissenschaftseinrichtungen ihre fast 10.000 jährlich in Wiley-Zeitschriften erscheinenden Fachartikel im Open Access unter einer freien Lizenz zu veröffentlichen." Vertrag zwischen DEAL und Wiley abgeschlossen, 15. Januar 2019 - https://bit.ly/2lHYyHi. Die Gebühr ist keine APC für das Open-Access-Publizieren alleine, sondern eine sogenannte PAR (publish\&read). Sie erlaubt zusätzlich das Lesen aller bei Wiley erscheinenden Zeitschriftenartikel. Bei Publikationen in bestehenden Wiley Open-AccessGold-Zeitschriften muss eine APC weitergezahlt werden; allerdings werden darauf $20 \%$ Rabatt gewährt. Vgl. Eckpunkte des Memorandum of Understanding mit Springer Nature, 22.8.2019 https://bit.ly/38gPLiQ.

611 Für 2020 basiert der PAR aber wohl auf „mindestens 9.500 Artikeln“, so die Pressemitteilung der MPI dazu: https://bit.ly/325KgQq.

612 So im Wiley-Vertrag genannt (FN 608). 
zierten Open-Access-Artikel von Autoren [...] aus den teilnahmeberechtigten deutschen Einrichtungen” (vgl. FN 608).

(2) Für jeden Artikel ist von der Einrichtung des publizierenden Autors eine sogenannte PAR-Gebühr (publish\&read) von $€ 2.750$ zu entrichten. Das PAR-Prinzip wurde schon früher verschiedentlich bei Verträgen mit Verlagen angewendet, aber jetzt bei DEAL im großen Stil.

(3) PAR ist keine traditionelle APC, sondern erlaubt jetzt den rund 700 teilnahmeberechtigten deutschen Wissenschaftseinrichtungen einen dauerhaften lesenden Zugriff auf die wissenschaftlichen Zeitschriften von Springer, Palgrave, Adis und Macmillan bzw. auf die von Wiley, aber nur für die, die in der Laufzeit des Vertrags erschienen sind. Einige wichtige Zeitschriften und Magazine sind zudem davon ausgeschlossen. ${ }^{613}$ Unklar ist, ob dieser lesende Zugriff davon abhängig ist, ob eine bestimmte Anzahl von PAR erreicht wird.

(4) Während der Verlagslaufzeit (also bis 2022 bzw. eventuell 2023) kann auch auf die sogenannten Backfiles bis zum Erscheinungsjahr 1997 zurückgegriffen werden. Es bleibt entsprechend wohl ein Zugriffsloch für die Jahre zwischen 1998 bis 2020 (der Beginn des Deal-Deals). Das sind allerdings die vor allen in STM/MINT-Fächern wichtigen, intensiv genutzten Jahrgänge. Fraglich ist, ob dieses Loch dann weiter über Subskriptionen geschlossen werden muss.

(5) In Ergänzung zu (1) können weitere Artikel nach Open-Access-Prinzipien in Wiley- bzw. in Springer Gold-Open Access- oder in hybriden Zeitschriften bzw. in BioMed Central publiziert werden. Für diese würde weiter das APC-Modell gelten. Allerdings wird für Institutionen, die bei DEAL beteiligt sind (derzeit ca. 700), für APC ein Rabatt von $20 \%$ von der PAR-Gebühr gewährt.

(6) Eine Nutzung nach Open Access-Prinzipien wird nach wie vor nur bei jenen Artikeln möglich sein, die unter einer freien Lizenz publiziert wurden, entweder in hybriden oder in reinen Open Access-Zeitschriften.

(7) Unklar ist, welche Folgen der unter (3) erwähnte lesende Zugriff haben wird. In der Urheberrechtsterminologie würde das bedeuten, dass das Ausdrucken und Abspeichern geschweige denn das öffentliche Zugänglichmachen dieser Materialien für Zwecke von Forschung und Lehre nicht gestattet wäre.

(8) Die jeweilige PAR-Gebühr ist aus den Budgets der Forschungseinrichtungen bzw. deren Bibliotheken zu entrichten, bei denen ein Autor einen Artikel nach Open Access-Prinzipen veröffentlichen will. Entsprechend werden dadurch publikationsintensive Einrichtungen stärker belastet als jene, bei denen weni-

613 Bei Springer z. B. "Nature and Nature branded subscription journals or purely professional journals as well as magazines, such as Scientific American or Spektrum der Wissenschaft”https://bit.ly/3743d9h. 
ger publiziert wird. Da über die PAR-Gebühren aber auch der gebührenfreie lesende Zugriff auf die kommerziellen Bestände erlaubt ist (nach den unter (3) und (4) angeführten Bedingungen), ist das sozusagen eine Solidaritätsaktion zugunsten aller anderen Nutzer aus den (bislang) 700 Einrichtungen. ${ }^{614}$

DEAL ist ein Schritt in Richtung des Ziels, die Bibliotheken vom bisherigen Subskriptionsmodell zu befreien. Insgesamt sind die jetzigen Vereinbarungen aber erst eine Annäherung an die (oben skizzierten) ursprünglich anvisierten Ziele:

(1) Das erste DEAL-Ziel, dauerhafter Vollzugriff aller DEAL-Partner auf alle publizierten Zeitschriften, wird durch den jetzigen Vertrag nicht in dem anvisierten Umfang erreicht.

(2) Angesichts der verschiedenen einschränkenden Bedingungen ist es fraglich, ob entsprechend dem dritten DEAL-Ziel ein einfaches, zukunftsorientiertes Berechnungsmodell entwickelt worden ist. Durch das UrhWissG hatte der Gesetzgeber in der UrhWissG-Reform 2017/18 das Prinzip der Individualerhebung und -vergütung für schrankenbasierte Nutzung erst einmal, auch wegen zu hohen Transaktionskosten aufgegeben. Nun kommt auf die Bibliotheken ein kompliziertes Verfahren für die Open Access-Beiträge in den Zeitschriften der Verlage zu.

(3) Ob entsprechend dem vierten DEAL-Ziel alle DEAL-Einrichtungen tatsächlich finanziell entlastet werden, bleibt noch offen. Für publikationsintensive Einrichtungen ist dies fraglich.

Problematisch ist, dass die Rückwirkung der Vereinbarung für das Lesen früher kommerziell publizierter Artikel auf die Laufzeit des Deals bzw. die Zeit bis 1997 beschränkt ist. Die Zeit zwischen 1998 bis 2020 bleibt - so müssen die Eckpunkte ausgelegt werden - offenbar ausgespart. Einige wichtige Zeitschriften bleiben vom freien „Lesen“ ausgenommen. Die Skepsis bleibt, ob durch die Vereinbarung mit der PAR-Gebühr Mittel für die Realisierung von Open-Access-Modellen aus der Wissenschaftsumgebung freigesetzt werden können. Kurz- und mittelfristig wird das eher nicht der Fall sein. Die Gefahr der weiteren Preisspirale ist nicht gebannt. Möglicherweise wird dadurch sogar das Marktmonopol der großen Verlagskonsortien verstärkt. Unterm Strich überwiegt aber der jetzige Erfolg von DEAL, der ja nicht das Ende sein muss. Trotz mancher Bedenken vor allem der viel zahlenden Vielpublizierer ist der jetzige DEAL mit Wiley und Springer Nature ein wichtiger Hinweis dafür, dass die umfassende Open-Access-Transformation unterwegs ist.

614 In dem Berechnungsmodell nach Publikationsaufkommen steckt einiges Konfliktpotenzial. Darauf hat der Verein der TU-Universitäten in Deutschland in einer Pressemitteilung „Open Access Transformation zum Erfolg führen“ vom 25.11.2019 hingewiesen - https://bit.ly/2YqpPgI. Die TU-Universitäten sehen in der Tat „höhere Kosten für die publikationsstarken Einrichtungen“. Aus Ausgleich wird ,im Sinne einer fairen Kostenverteilung“ erwogen, die weniger publikationsintensiven Hochschulen, die aber auch an den Vorteilen von DEAL partizipieren, ,an der Finanzierung zu beteiligen, z. B. in Form einer reading fee“. 
Das Open Access inhärente Prinzip der Nutzungsfreiheit gewinnt durch diese gemeinsame Aktion der deutschen Wissenschaftsorganisationen und den Wissenschaftseinrichtungen innerhalb und außerhalb der Hochschulen öffentliche Anerkennung. Weltweit wird diese Entwicklung durch Initiativen wie OA2020 vorangetrieben (s. zu Beginn dieses Abschnitts).

\subsection{Open-Access-Märkte für Bücher}

Die Transformation der Wissenschaftsmärkte in Richtung Open-Access-Publizieren hat schon jetzt auch Auswirkungen auf Produktion und Vertrieb größerer Werke wie Monographien, Sammelbände, Proceedings ${ }^{615}$, Lehrbücher, Festschriften etc. DOAB, das Directory for Open Access Books, ${ }^{616}$ weist Anfang Februar 202026.841 wissenschaftliche peer-reviewed Bücher nach, veröffentlicht von 370 Verlagen. OAPEN ${ }^{617}$ weist Open-Access-Bücher überwiegend aus den Sozial- und Geisteswissenschaften nach und unterstützt dessen Produktion.

Dabei ist davon auszugehen, dass die Transformation der Wissenschaftsmärkte auch für das Publizieren von Büchern in Richtung Open Access geht. Dafür nur ein Hinweis auf SpringerOpen, welches seine Plattform SpringerLink zur Bereitstellung von Open-Access-Büchern nutzt. Springer ermuntert Autoren zur Open-AccessPublikation ihrer Bücher mit dem Hinweis auf größere Sichtbarkeit und Nutzung ihrer Werke:

Tour open access academic books and chapters on average receive seven times more downloads, $50 \%$ more citations, and ten times more online mentions compared to non-open access books ${ }^{\star}{ }^{618}$

Daneben behalten die Autoren gänzlich ihre Urheberrechte, und die Erstellung der elektronischen Versionen erfolgt, so SpringerOpen, mit der gleichen redaktionellen Sorgfalt, wie sie bei den gedruckten Büchern üblich sind. Die Finanzierungsmodelle

615 Vor allem bei den Proceedings von Konferenzen ist Entwicklung zu den Open-Access-Büchern deutlich zu erkennen: Diese werden häufig von den Fachgesellschaften organisiert und finanziert. Die Teilnahme an den i.d. R. kostenpflichtigen Konferenzen sichert den Teilnehmern (und damit indirekt auch ihren Kollegen) die freie Nutzung dieser Arbeiten. Auch bei diesem Publikationsbereich werden die Verlage aktiv und bieten z. B. eigene Proceedings Software an (vgl. die von Cern bereitgestellte Liste von Verlagen, die solche Conference-Proceedings-Software anbieten - Projekt indico - https://getindico.io/).

616 DOAB - https://www.doabooks.org/.

617 OAPEN - https://www.oapen.org/home.

618 SpringerOpen - https://www.springeropen.com/books. 
für Open-Access-Gold-Bücher sind aber nicht durchgängig transparent. Im hybriden Modell könnte die Finanzierung der Open-Access-Gold-Bücher durch den Verkauf der nach wie vor gedruckten Exemplare gesichert werden. Zur Anwendung kommen aber auch Online-Nutzungsmodelle, bei denen nur einzelne Kapitel gegen Zahlung online verfügbar gemacht werden. Das scheint eher das Modell für Textsorten wie Handbücher zu sein, bei denen die einzelnen Kapitel selbständige Texte sind.

Derzeit verlangen die meisten Verlage von den Autoren für die Freistellung der entsprechenden Open-Access-Gold-Version, dass sie auf irgendeine Weise für die Produktions- und Bereitstellungskosten für diese Version aufkommen. Die bei Zeitschriftenartikel gebräuchliche APC wird auf Bücher übertragen. Üblich sind hohe 4-stellige Euro-Beträge, über deren genaue Höhe wohl von Fall zu Fall entschieden wird. Diese Gebühr wird in der Erwartung erhoben - ohne dass es dafür empirische Belege gibt -, dass durch die Gold-Version der Verkauf der gedruckten Version eingeschränkt wird.

Es kommen aber auch andere Modelle zum Einsatz. De Gruyter z. B. experimentiert, neben der auch der hier angewendeten Open-Access-Gold-Publikation, mit verschiedene Verfahren für die E-Verfügbarkeit. So schließt de Gruyter mit einzelnen Bibliotheken Nutzungsverträge für den Online-Zugriff auf die Web-Plattform „De Gruyter“ ab. ${ }^{619}$ Das erlaubt nicht unbedingt in jedem Fall eine Nutzung nach Open-Access-Prinzipien, schließt diese aber auch nicht prinzipiell aus. Die Nutzung ist über genau spezifizierte Lizenzbedingungen geregelt. ${ }^{620}$ Weitergehend ist ein Projekt, das derzeit (Oktober 2019) De Gruyter mit der Staats- und Universitätsbibliothek Göttingen begonnen hat. ${ }^{621}$ Dieses Projekt soll die Open-Access-Transformation von Büchern in den Geisteswissenschaften befördern. Das bisherige Lizenzierungsmodell soll durch ein Open-Access-Produktionsmodell ersetzt werden. Die an dem Projekt teilnehmenden Bibliotheken finanzieren gemeinsam die Veröffentlichung von Monographien und Sammelbänden nach Open-Access-Prinzipien. Die Analogie zu den bei Zeitschriften angestrebten und z. B. über DEAL schon teilweise vereinbarten Transformationsmodellen (dort vom Subskriptionsmodell zum Erstellungsmodell, vgl. 14.8) ist unverkennbar. Die Refinanzierung der Ausgaben der Verlage geschieht nicht über die Nutzung, sondern über die Einnahmen aus der Vergütung für die Produktion und Bereitstellung der Informationsobjekte, welche die Öffentlichkeit derzeit bereit ist zu erbringen. Nutzungsfreiheit wird auch das Prinzip für größere Werke wie Bücher. Auch das sollte Folgen für die auf kleinteilige Nutzung abzielenden urheberrechtlichen Schrankenbestimmungen haben. Die

619 Z.B. De Gruyter Online für die Universität Hildesheim - https://bit.ly/32lsWGQ.

620 Eine Übersicht über die Lizenzbedingungen verschiedener Verlage/Anbieter von der Universität Viadrina Frankurt/Oder: https://bit.ly/33xxD1X.

621 de Gruyter/Bibliothek Göttingen Open Access Projekt - https://bit.ly/2Cjul6s. 
Beschränkungen der Nutzung auf festgelegte Prozentsätze des Gesamtumfangs wird im Open-Access-Gold Ansatz-überflüssig.

\subsection{Open Access wird Default des wissenschaftlichen Publizierens}

Die Entwicklung auf den Zeitschriftenmärkten (und noch etwas langsamer auch auf den Buchmärkten) legt es nahe: Es ist heute keine gewagte These mehr, dass das Open-Access-Publizieren das allgemeine Paradigma sozusagen der Default des Publizierens in der Wissenschaft wird. ${ }^{622}$ Schon in der Strategie des BMBF zu Open Access in Deutschland von 2016 hieß es: „Open Access als Standard des wissenschaftlichen Publizierens etablieren““.623 Open Access zu realisieren, ist auch seit mehr als 12 Jahren fester Bestandteil der EU-Politik - dokumentiert in zahlreichen Stellungnahmen vor allem der EU-Kommission, aber auch des EU Rats. ${ }^{624}$ Dabei ist Open Access nur ein Teil des umfassend allgemein gewordenen Open-Prinzips, vor allem mit Blick auf Open Science. ${ }^{625}$ Auch der EU Rat hatte

622 In Deutschland informiert laufend der Nationale Open-Access-Kontaktpunkt OA2020-DE zum Online-/Open-Access-Publizieren - https://oa2020-de.org/.

623 BMBF Open Access in Deutschland - https://bit.ly/2k6hAXc. Einige Allianzorganisationen hatten darin schon klare Ziele bis 2020 formuliert: Die Helmholtz-Gemeinschaft hat im April 2016 erklärt, ihre Bemühungen zu Open Access zu quantifizieren. Bis 2020 sollen mindestens 60 Prozent der Publikationen Open Access verfügbar sein, im Jahr 2025100 Prozent. Die Fraunhofer-Gesellschaft strebt bis 2020 einen Open Access-Anteil von 50 Prozent ihrer Publikationen an.

624 EU-Kommission; „Policies on open access to scientific research results should apply to all research that receives public funds" und zwar "as soon as possible, preferably immediately and in any case no later than six months after the date of publication, and twelve months for social sciences and humanities" - Communication from the Commission ... Towards better access to scientific information: Boosting the benefits of public investments in research. $\operatorname{COM(2012)~} 401$ final, 17.2.2012 - https://bit.ly/1IP1hBg. Aber schon 2007 hatte sich die EU-Kommission mit Open Access als Teil der „Forschungspolitik der Gemeinschaft zur Maximierung des sozioökonomischen Nutzens von Forschungs- und Entwicklungsbestrebungen für das Allgemeinwohl” beschäftigt - Mitteilung der Kommission .... über wissenschaftliche Informationen im Digitalzeitalter: Zugang, Verbreitung und Bewahrung \{SEC(2007)181\} - KOM(2007) 56 vom 14.2.2007 - https://bit.ly/35V4ZI9.

625 Vgl. die Artikel im Sonderheft zu “Open Science” vom 15.08.2017 in der Zeitschrift für Technologiefolgenabschätzung in Theorie und Praxis (TATuP) Bd. 26, Nr. 1-2, 2017; insbesondere die Artikel von Werner Reichmann: Open Access zwischen sozialen Strukturen und Wissenskulturen; Ulrich Riehm, Michael Nentwich: Open Science aus Perspektive der Technikfolgenabschätzung; Johann Jakob Häußermann, Marie Heidingsfelder: Offen, verantwortlich und verantwortlich offen; Heidemarie Hanekop: Umwandlung wissenschaftlicher Journale in Gold Open Access. Chance 
schon 2016 Open Access allgemein in die politische Zielsetzung eines Übergangs in ein umfassendes Open-Science-System eingebettet. ${ }^{626}$ Die europäischen Organisationen zur Wissenschaftsförderung bewegen sich immer mehr in Richtung eines umfassenden Open-Access-Publizierens (vgl. die Initiativen von OA2020 und von cOAlition Plan S). Auch die DFG hat eine öffentliche Unterstützung für den Plan S der cOAlition signalisiert. Die DFG forcierte $2018 \mathrm{ihr}$ Engagement zugunsten eines verbindlichen Open Access und fordert die von ihr geförderten Wissenschaftler auf,

ihre Ergebnisse entweder auf dem sogenannten „Goldenen Weg“ (sofortige Open Access-Veröffentlichung) oder auf dem „Grünen Weg“ (Veröffentlichung in Open Access-Repositorien zusätzlich zur Veröffentlichung nach dem Subskriptionsmodell) öffentlich zugänglich zu machen. ${ }^{627}$

Die DFG unterstützt die von ihr geförderten Wissenschaftler finanziell, ihre „Forschungsergebnisse auf Open-Access-Basis kostenfrei im Internet zur Verfügung zu stellen” und fordert sie auf, „einen offenen Zugang zu geförderten Publikationen zu ermöglichen"(ebda.). Auf eine Verpflichtung hat sie sich, ähnlich wie auch bei der Zweitverwertung nach §38 Abs. 4 UrhG (vgl. 11.3), (noch) nicht verständigen können - anders als z. B. in der Schweiz, z. B. durch den Schweizerischen Nationalfonds ${ }^{628}$ sowie durch Swiss National Science Foundation (SNSF) ${ }^{629}$ und in Österreich durch den Österreichischen Nationalfonds. ${ }^{630}$ Im von Stevan Harnad organisierten Registry of Open Access Repository Mandates and Policies (ROARMAP) sind weltweit 86 Förderorganisationen nachgewiesen, die OA mandatorisch verlangen (dabei keine einzige aus Deutschland) - darüber hinaus annähernd 1000 Forschungseinrichtungen und Verbände, die entweder ein mandatorisches Open-Access-Publizieren verpflichtend machen (required) oder dies dringend nahelegen (requested).

Die Öffentlichkeit ist derzeit bereit, den Verlagen ihre Produktions- und Distributionsleistungen für Zeitschriftenartikel finanziell in einem solchen Umfang zu entgelten, dass die Nutzung der publizierten Materialien nach Open-Access-

oder Risiko für die Wissenschaft?; Werner Reichmann: Open Science zwischen sozialen Strukturen und Wissenskulturen. Eine wissenschaftssoziologische Erweiterung.

626 (Council 2016) EU Council 8791/16 vom 17.5.2016 - https://bit.ly/25xBYxb.

627 In der Stellungnahme der DFG zur Gründung von „COAlition S“ zur Unterstützung von Open Access. Information für die Wissenschaft Nr. 56 | 4. September 2018 - https://bit.ly/2Humea5.

628 Schweizer Nationalfonds: Auf dem Weg zu $100 \%$ : Steigerung bei Open-Access-Publikationen notwendig - https://bit.ly/31hxLAY. Kritisch dazu Michael Baumann: Das neue Urheberrecht bremst Open Access. Horizonte 8.3.2018 - https://bit.ly/370D9vD.

629 SFNS: "The rules of the SNSF require grantees to make their research results available in an Open Access publication.” Overview of SNSF Guidelines on Open Access - https://bit.ly/30CkAKN. Das gilt gleichermaßen für Zeitschriftenartikel wie auch für Bücher.

630 Österreichischen Nationalfonds. Open Access Policy -https://bit.ly/2KVTucg. 
Prinzipien frei ist. Das klassische Modell der Verlagswirtschaft - erst einmal in finanzielle Vorleistung zu treten, um dann durch Verkauf oder Lizenz den Return of Investment einschließlich der Gewinne zu sichern - dreht sich im kommerziellen Publizieren fast vollständig um. Der Return of investment ist quasi vorfinanziert. Die Nutzung produziert keine weiteren Einnahmen. Man könnte der Ansicht sein, dass es der Wissenschaft letztlich egal ist, wer diese Freiheit garantiert - Bibliotheken und andere öffentliche Informationsvermittlung-/Kultur-Einrichtungen oder die Verlagswirtschaft, die das finanziert gesichert sehen will. Ob allerdings das Verfahren, das kommerzielle Open-Access-Publizieren öffentlich zu finanzieren, tatsächlich nachhaltig und aus volkswirtschaftlicher Sicht sinnvoll ist, ist noch nicht ausdiskutiert. Auch ist es noch nicht abschließend durchgerechnet, welche Open-Access-Verfahren ökonomischer preisgünstiger sind - die in öffentlichen Umgebungen organisierten oder die in kommerziellen Umgebungen. Das Argument, dass die öffentlichen Einrichtungen für ihre Publikationsleistungen keinen Gewinn erzielen müssen bzw. es auch nicht dürfen, ist zwar stark, aber vielleicht nicht ausreichend. Die Gefahr besteht, dass durch die Umschichtung von öffentlichen Geldern zugunsten der Finanzierung des kommerziellen Open Access-Publizierens die Budgets der Bibliotheken stark eingeschränkt würden. Schließlich ist ungeklärt, wie die Etats mit den entstehenden Preissteigerungen umgehen sollen, insbesondere an den publikationsstarken Universitäten. Ob all das durch DEAL vermieden werden kann, muss sich zeigen. Bibliotheken sind mehr denn je, auch unabhängig von der Bereitstellung publizierten Wissens, sichere soziale Orte der Kommunikation und der professionellen Beratung und Qualitätssicherung. Das sollte erhalten bleiben.

\subsection{Perspektiven für neue kommerzielle Informationsmärkte}

Die Transformation der Informationsmärkte hat sich bislang in erster Linie auf das Publizieren und hier vor allem auf die Zeitschriftenmärkte bezogen. Derzeit scheint es gesichert, dass durch die finanzielle Unterstützung der Öffentlichkeit die kommerziellen Verwerter erfolgreich die Transformationsphase überstehen und auf den Märkten weiter aktiv sein können. Das mag eine sinnvolle Investition der Öffentlichkeit gewesen sein, um auch den Zugriff auf die vielen Millionen schon in kommerziellen Umgebungen produzierten und weiter benötigten Publikationen zu sichern und die Bibliotheken von den belastenden Subskriptionslasten für Bündel von Zeitschriften zu befreien. Das muss aber nicht auf Dauer so sein. Letztlich handelt es sich auch bei Initiativen wie DEAL um eine Subventionspolitik, die, wie viele andere Subventionen, Innovationen auf den Märkten eher behindern oder verzögern könnte. Zudem ist die Entwicklung, dass Wissenschaft aus sich heraus 
und in Zusammenarbeit mit den ihnen zuarbeitenden Bibliotheken oder anderen öffentlich finanzierten Dienstleistern die Aufgabe des Open-Access-Publizierens organisiert, weiter eine realistische Perspektive. In 14.5 wurde schon (Ziegler 2019) zitiert, dass Wissenschaftlerinnen und Wissenschaftler die Arbeit der Verlage weitgehend selbstständig und unabhängig übernehmen" könnten. In FN 582 wurde schon auf verfügbare Endnutzersoftware z. B. zum Erstellen von Open-Access-Zeitschriften verwiesen; ebenso dort auf die vielfältigen Aktivitäten zur Unterstützung für die Produktion und Bereitstellung von Open-Access-Büchern. An vielen Universitäten entstehen auf OA ausgerichtete Universitätsverlage. Derzeit haben sich 29 dieser Verlage in der AG Universitätsverlage zusammengeschlossen. ${ }^{631}$ Auch die Association of European University Presses (AEUP) hat 2018 Leitlinien für die Arbeit dieser Verlage herausgegeben, davon wird besonders unter (5) die Ausrichtung auf Open Access hervorgehoben:

Access to scholarly communication needs to be as free and inclusive as possible to allow society benefit fully from research. Open Access and Open Science are the right means to reach this objective. ${ }^{632}$

Das ist ein deutlicher Hinweis darauf, dass alle Leistungen von Open-Initiativen nicht exklusiv auf Bildung und Wissenschaft ausgerichtet sein dürfen, sondern der Öffentlichkeit allgemein frei nutzbar gemacht werden sollten. Was die Öffentlichkeit finanziert, soll i. d. R. auch öffentlich frei genutzt werden können. Ausnahmen von dieser Regel bedürfen einer besonderen Rechtfertigung (z. B. über Datenschutz, Sicherung der Privatheit).

Die Entwicklung deutet darauf hin, dass sich konkurrierende offene und kommerzielle Open-Access-Märkte entwickeln können. Da Open-Access-Aktivitäten aus der Wissenschaftsumgebung nicht auf Gewinnmaximierung ausgerichtet sind, könnten diese vermutlich kostengünstiger arbeiten als die auf Gewinn angewiesenen kommerziellen Verlage. Was sich wie gesamtvolkswirtschaftlich rechnet, bedarf jedoch gründlicher Evaluierung. Es wäre zu wünschen, wenn die Öffentlichkeit diese Aktivitäten im gleichen Umfang auch finanziell unterstützt, wie sie es

631 Prinzipien der AG Universitätsverlage: „Die Mitglieder sind an eine Forschungseinrichtung angebunden, spiegeln im Verlagsprogramm das Profil ihrer Einrichtung wider, setzen Open Access und eine wissenschaftsfreundliche Rechtepolitik um, treffen Maßnahmen zur Gewährleistung hoher wissenschaftlicher und formaler Qualität, verfolgen keine Gewinnmaximierung, arbeiten transparent und kollegial zusammen.“ - https://bit.ly/2nQhcxL. Dafür wurden Qualitätsstandards für Open-Access-Monographien und Sammelwerke erarbeitet - https://bit.ly/2mkKvbd.

632 Association of European University Presses (AEUP): Seven Statements on European University Presses - https://bit.ly/2lEqsEn. Eine Anpassung dieser Leitlinien der ag universitätsverlage https://bit.ly/2lCdAi1. 
derzeit für die kommerziellen Verlage tut. Das gilt ganz besonders auch für die Open Access-Initiativen, die auf Lehre und das Lernen ausgerichtet sind. ${ }^{633}$ Dafür hat sich die Sammelbezeichnung „Open Educational Resources“ (OER) durchgesetzt. OER-Aktivitäten in Deutschland werden koordiniert durch die Informationsstelle OER, eingerichtet am Deutschen Institut für Pädagogische Forschung (DIPF). ${ }^{634}$ International werden die OER-Initiativen durch das Open Education Consortium koordiniert. Derzeit sind 243 Institutionen in 44 Ländern daran beteiligt. Das Ziel des Consortium ist es,

to build capacity to find, reuse, create and share Open Educational Resources (OER), develop open policy, create sustainable open education models, and enable international collaboration and innovation.

Einiges deutet darauf hin, dass das Publizieren von Wissensobjekten aus der Wissenschaft zwar weiter noch eine Weile das Kerngeschäft der Verlage bleibt, dass sich aber Verlage zunehmend auch als Dienstleister für Bildung und Wissenschaft neu bestimmen. Elsevier, weiter der größte Akteur auf den Publikationsmärkten, hat dafür das Etikett Research Intelligence Provider (REP) ins Spiel gebracht. Zielgruppen von REP sind

\begin{abstract}
„Universitäten, Forschungsinstituten, Investoren und Entscheidungsträgern an, die sich auf Research Intelligence verlassen, um Probleme zu lösen und ihre Fähigkeiten zu erweitern und so Ihre Leistung in der Forschung zu verbessern“:635 Für Forschungsinstitute wird ein „umfassendes Portfolio an Forschungsmanagementlösungen an[geboten], die einen klaren Überblick über die Forschungsaktivitäten“ geben. Investoren können unterstützt werden, „die Effektivität der Forschungsprojekte, die sie unterstützen, zu messen, Reviewer zu finden und Trends in den wichtigsten Forschungsbereichen zu identifizieren." Politischen Entscheidungsträger werden ,die Mittel zur Verfügung [gestellt], mit denen [...] die Leistung der Forschung sowie Trends in Bezug auf die Zusammenarbeit bewertet werden, die Wirksamkeit von Forschungsrichtlinien bestimmt sowie Exzellenz in der Forschung demonstriert [werden] können“.
\end{abstract}

(Herb 2018) hat diese Tendenz ausführlich analysiert ${ }^{636}$ und dabei als zentrale Geschäftsfelder ausgemacht: Bibliometrie, Benchmarking, Forschungsinformation,

633 Für einen Überblick über die OER-Entwicklung in Deutschland und Vorschlägen für deren Förderung vgl. (Mollenhauer /Blees/Rittberger 2017) Open Educational Resources (OER) in Deutschland fördern.

634 OERinfo Informationsstelle Open Educational Resources - https://bit.ly/2h6yCj4.

635 Elsevier: Wer verwendet Research Intelligence Solutions? (Deutsche Version von REP) https://bit.ly/2nQYxSn.

636 (Herb 2019) Überwachungskapitalismus und Wissenschaftssteuerung” - https://bit.ly/ 2Nw4r5p. 
Reference Management, Medienmonitoring, Forschungsdaten-Management, Disziplinäre und institutionelle Open-Access-Repositories, Altmetrics. Herb hat aber auch REP als Gefahr interpretiert, nämlich dass über die Analyse großer Datenmengen, die aus der Wissenschaft produziert und z. B. durch SCOPUS/Elsevier ausgewertet werden, Kontrollmechanismen entwickelt werden, die über Kriterien wie Steuerung, Effizienz und Rentabilität wissenschaftliche Autonomie aussetzen können. Das ist die dystopische Sicht dieser REP-Entwicklung. ${ }^{637}$ Durch REP könnten aber auch Produkte und Dienstleistungen mit informationellen Mehrwertleistungen erstellt und auch neue Messverfahren (Altmetrics) entwickelt werden, die die Abhängigkeit von dem Impact Factor auflösen könnten. Das Kriterium für informationelle Mehrwerte sollte aber immer der Nutzungsmehrwert für die Akteure in Bildung und Wissenschaft sein. Es ist zu erwarten, dass auch die anderen großen Verlagskonsortien wie z. B. Wiley, Springer Nature, aber auch die alternativen Content Provider wie Google, Facebook, aber auch Universalanbieter wie Amazon diese auf Big Data beruhenden Produkte und Dienstleistungen aufbauen werden.

Gesamtfazit von Kapitel 14. Die erkennbare Transformation des bisherigen kommerziellen Publizierens in Open-Access-Publizieren bestätigt, dass die Märkte sich nicht gegen sich entwickelnde Leitideen behaupten können bzw. dass sie diese aufgreifen. Das kann auch als Bestätigung der These gewertet werden, dass die beiden Informationsmärkte, die proprietären und offenen, sich nicht gegenseitig ausschließen müssen - allerdings unter der Bedingung, dass die Leitidee des offenen freien Umgangs mit Wissen und Information auf beiden Märkten akzeptiert wird. Das scheint der Fall zu sein und bestätigt die auf den ersten Blick paradoxe These, dass die kommerziellen Akteure auf den wissenschaftlichen Informationsmärkten sich nur dann auf diesen behaupten können, wenn sie den Zugang und die Nutzung ihrer Informationsprodukte so frei wie möglich machen. Allerdings ist es durchaus noch nicht entschieden, ob eventuell die aus der Wissenschaft selbst entwickelten Open-Access-Modelle letzten Endes die bessere und sogar preiswertere Lösung für die Informationsversorgung von Bildung und Wissenschaft sein könnten. Inwieweit die kommerzielle Verlagstätigkeit sich in Zukunft in erster Linie auf die in Abschnitt 14.11 angedeuteten neuen, über das Publizieren

637 (Herb 2018) Zwangsehen und Bastarde): „So ergeben sich durch REP ... umfassende Fragen nach Datenschutz und Persönlichkeitsrechten [Letzteres im Sinne des GG, nicht verstanden als Urheberpersönlichkeitsrechte - RK]. Die heute mögliche, in unvorhersehbarer Weise exakte Identifikation eines einzelnen Wissenschaftlers in all seinen beruflichen Schattierungen und Aktivitäten erlauben zudem eine umfassende Kontrolle, ein unaufhörliches Benchmarking und ein anhaltendes Bewerten der Forschung z. B. hinsichtlich der Berücksichtigung ihrer Anschlussfähigkeit an die (internationalen) Trends.“, S. 87. 
hinausgehenden Produkte und Dienstleistungen konzentrieren wird, wird sich zeigen. Einiges spricht aber dafür, dass sich grundlegend neue Geschäftsmodelle entwickeln werden. Ob die für diese Modelle erforderlichen Daten, die aus der Wissenschaft stammen und für neuen Geschäftsfelder verwendet werden, auch über das Urheberrecht oder eher über den Datenschutz reguliert werden sollen, ist eine offene Frage. Ebenso wird kontrovers diskutiert, ob durch Big Data die bisherige Einschätzung revidiert werden soll, dass Daten und Fakten nicht über das Urheberrecht geschützt und nicht reguliert werden. Eine solche Erweiterung des Urheberrechts wäre sicher nicht im Interesse von Bildung und Wissenschaft.

In Kap. 4 wurde die Priorität von informationsethisch entwickelten Leitideen gegenüber den anderen Regulierungsinstanzen eingefordert. Nachdem auch die Märkte diese Ideen aufgreifen, ist es auch für das Urheberrecht an der Zeit, der Leitidee des offenen freien Umgangs mit Wissen und Information mit der Konsequenz der Nutzungsfreiheit Rechnung zu tragen. Darauf wird im abschließenden Kapitel eingegangen. 
